\title{
EXTREMES OF REGULARLY VARYING LÉVY-DRIVEN MIXED MOVING AVERAGE PROCESSES
}

\author{
VICKY FASEN, ${ }^{*}$ Munich University of Technology
}

\begin{abstract}
In this paper, we study the extremal behavior of stationary mixed moving average processes of the form $Y(t)=\int_{\mathbb{R}_{+} \times \mathbb{R}} f(r, t-s) \mathrm{d} \Lambda(r, s), t \in \mathbb{R}$, where $f$ is a deterministic function and $\Lambda$ is an infinitely divisible, independently scattered random measure whose underlying driving Lévy process is regularly varying. We give sufficient conditions for the stationarity of $Y$ and compute the tail behavior of certain functionals of $Y$. The extremal behavior is modeled by marked point processes on a discrete-time skeleton chosen properly by the jump times of the underlying driving Lévy process and the extremes of the kernel function. The sequences of marked point processes converge weakly to a cluster Poisson random measure and reflect extremes of $Y$ at a high level. We also show convergence of the partial maxima to the Fréchet distribution. Our models and results cover short- and long-range dependence regimes.
\end{abstract}

Keywords: Continuous-time moving average process; extreme-value theory; independently scattered random measure; long-range dependence; marked point process; point process; regular variation; shot noise process; supOU process

2000 Mathematics Subject Classification: Primary 60G70

Secondary 60F05; 60G10; 60G55

\section{Introduction}

In this paper, we investigate the extremal behavior of a stationary, continuous-time mixed moving average (MA) process of the form

$$
Y(t)=\int_{\mathbb{R}_{+} \times \mathbb{R}} f(r, t-s) \mathrm{d} \Lambda(r, s), \quad t \in \mathbb{R},
$$

where the kernel function $f: \mathbb{R}_{+} \times \mathbb{R} \rightarrow \mathbb{R}$ is measurable and $\Lambda$ is an infinitely divisible, independently scattered random measure (IDISRM). Recall the definition of an IDISRM on $\mathbb{R}_{+} \times \mathbb{R}$ : let $\mathcal{A}$ be a $\delta$-ring (i.e. a ring closed under countable intersections) of $\mathbb{R}_{+} \times \mathbb{R}$ such that there exists an increasing sequence $\left\{S_{n}\right\}_{n \in \mathbb{N}}$ of sets in $\mathcal{A}$ with $\bigcup_{n=1}^{\infty} S_{n}=\mathbb{R}_{+} \times \mathbb{R}$. Moreover, let $\Lambda=\{\Lambda(A), A \in \mathcal{A}\}$ be a real-valued stochastic process defined on some probability space. We call $\Lambda$ an independently scattered random measure if, for every sequence $\left\{A_{n}\right\}_{n \in \mathbb{N}}$ of disjoint sets in $\mathcal{A}$, the random variables (RVs) $\Lambda\left(A_{n}\right), n \in \mathbb{N}$, are independent and, if $\bigcup_{n=1}^{\infty} A_{n} \in \mathcal{A}$, then $\Lambda\left(\bigcup_{n=1}^{\infty} A_{n}\right)=\sum_{n=1}^{\infty} \Lambda\left(A_{n}\right)$ almost surely (a.s.). We call a random measure infinitely divisible (ID) if $\Lambda(A)$ is ID for every $A \in \mathcal{A}$. The reader is referred to [29], [35], and [22] for more details on IDISRMs and integrals of the type shown in (1.1).

In the following, we consider only IDISRMs for which the characteristic function of $\Lambda(A)$ has the representation $\mathrm{E}[\exp (\mathrm{i} u \Lambda(A))]=\exp (\lambda(A) \psi(u))$ for $u \in \mathbb{R}$ and $A \in \mathcal{A}$. Throughout

Received 11 March 2005; revision received 18 May 2005.

* Postal address: Graduate Program in Applied Algorithmic Mathematics, Centre for Mathematical Sciences, Munich University of Technology, D-85747 Garching, Germany. Email address: fasen@ma.tum.de 
the paper, we assume that there exists a probability measure $\pi$ on $\mathbb{R}_{+}$such that $\lambda(\mathrm{d} \omega)=$ $\pi(\mathrm{d} r) \times \mathrm{d} t$ for $\omega=(r, t) \in \mathbb{R}_{+} \times \mathbb{R}$. Moreover, $\psi$ is the cumulant generating function of a Lévy process, with

$$
\psi(u)=\mathrm{i} u m-\frac{1}{2} u^{2} \sigma^{2}+\int_{\mathbb{R}}\left(\mathrm{e}^{\mathrm{i} u x}-1-\mathrm{i} u h(x)\right) v(\mathrm{~d} x) \quad \text { for } u \in \mathbb{R},
$$

where $h(x)=1_{[-1,1]}(x)$ is an indicator function. The quantity $\left(m, \sigma^{2}, v, \pi\right)$ is called the generating quadruple of the IDISRM $\Lambda$. Here $m \in \mathbb{R}, \sigma^{2} \geq 0$, and $v$ is the Lévy measure on $\mathbb{R}$, satisfying $v(\{0\})=0$ and $\int_{\mathbb{R}}\left(1 \wedge|x|^{2}\right) v(\mathrm{~d} x)<\infty$. We denote by $L=\{L(t)\}_{t \geq 0}$ the underlying driving Lévy process, with

$$
L(t)=\Lambda\left(\mathbb{R}_{+} \times[0, t]\right), \quad t \geq 0,
$$

and generating triplet $\left(m, \sigma^{2}, v\right)$.

Typical examples of mixed MA processes are the superpositions of Ornstein-Uhlenbeck (supOU) processes studied by Barndorff-Nielsen [2] (see Example 3.1, below), which are used in stochastic volatility modeling. If $f(r, s)$ is independent of $r$, i.e. $f(r, s)=\tilde{f}(s)$ for every $r \in \mathbb{R}_{+}$and $s \in \mathbb{R}$, and $\tilde{f}: \mathbb{R} \rightarrow \mathbb{R}$ is measurable, then we interpret $Y$ in (1.1) as the classical Lévy-driven MA process

$$
Y(t)=\int_{\mathbb{R}} f(t-s) \mathrm{d} L(s), \quad t \in \mathbb{R},
$$

where we have used the original symbol $f$ for the kernel function $\tilde{f}$. This class of process includes continuous-time autoregressive MA processes and fractionally integrated continuoustime autoregressive MA processes (see [9]) and stochastic delay equations (see [17]).

In the present paper, we investigate regularly varying Lévy-driven mixed MA processes with respect to their extremal behavior. We present the precise conditions below. For details on extreme-value theory, we refer the reader to the monograph [14]. We shall use the following standard notation: $\overline{\mathbb{R}}=\mathbb{R} \cup\{-\infty\} \cup\{\infty\}, \mathbb{R}_{+}=(0, \infty), \stackrel{\mathrm{w}}{\rightarrow}$ ' denotes weak convergence, and $\stackrel{\mathrm{v}}{\rightarrow}$ ' denotes vague convergence. For real functions $g$ and $h$, we write $g(t) \sim h(t)$ as $t \rightarrow \infty$ if $g(t) / h(t) \rightarrow 1$ as $t \rightarrow \infty$. For a set $A, \mathscr{B}(A)$ is the Borel $\sigma$-algebra of $A$.

Condition 1.1. The marginal distribution $L$ (1) of the underlying driving Lévy process $L$ in (1.2) is regularly varying of index $\alpha$ for some $\alpha>0$, i.e. there exists a sequence of constants $a_{n}$, $n \in \mathbb{N}$, such that $a_{n}>0, a_{n} \uparrow \infty$, and

$$
n \mathrm{P}\left(a_{n}^{-1} L(1) \in \cdot\right) \stackrel{\mathrm{v}}{\rightarrow} \sigma(\cdot) \quad \text { on } \mathcal{B}(\overline{\mathbb{R}} \backslash\{0\}), \quad \text { as } n \rightarrow \infty,
$$

where, for some $p \in[0,1]$ and $q=1-p$,

$$
\sigma(\mathrm{d} x)=p \alpha x^{-\alpha-1} 1_{(0, \infty)}(x) \mathrm{d} x+q \alpha(-x)^{-\alpha-1} 1_{(-\infty, 0)}(x) \mathrm{d} x .
$$

Regularly varying distribution functions (DFs) include, in particular, the stable, Pareto, loggamma, and Burr distributions. Notice that $\mathrm{E}\left[|L(1)|^{\delta}\right]<\infty$ for $\delta<\alpha$ and $\mathrm{E}\left[|L(1)|^{\delta}\right]=\infty$ for $\delta>\alpha$.

To study the extremal behavior of $Y$, we will impose the following condition in Section 4. We define

$$
\mathbb{L}^{\delta}(\pi):=\left\{f: \mathbb{R}_{+} \times \mathbb{R} \rightarrow \mathbb{R} \text { measurable, } \int_{\mathbb{R}_{+}} \int_{\mathbb{R}}|f(r, s)|^{\delta} \mathrm{d} s \pi(\mathrm{d} r)<\infty\right\}
$$

for $\delta>0$. If $f(r, s)$ is independent of $r$, we write $f \in \mathbb{L}^{\delta}$ instead of $f \in \mathbb{L}^{\delta}(\pi)$. 
Condition 1.2. Let $Y$ in (1.1) be a stationary, measurable, and separable ID process and let the underlying driving Lévy process $L$ in (1.2) satisfy Condition 1.1. Let the kernel function be $f \in \mathbb{L}^{\delta}(\pi)$ for some $\delta<\alpha$, or let L(1) be $\alpha$-stable and let $f \in \mathbb{L}^{\alpha}(\pi)$. In both cases, assume that

$$
f^{+}:=\sup _{(r, t) \in \mathbb{R}_{+} \times \mathbb{R}} f^{+}(r, t)<\infty \text { and } f^{-}:=\sup _{(r, t) \in \mathbb{R}_{+} \times \mathbb{R}} f^{-}(r, t)<\infty,
$$

where $f^{+}(r, t):=\max \{f(r, t), 0\}$ and $f^{-}(r, t):=\max \{-f(r, t), 0\}$. Furthermore, let

$$
\int_{\mathbb{R}_{+}} \int_{\mathbb{R}}\left(p\left(f^{+}(r, s)\right)^{\alpha}+q\left(f^{-}(r, s)\right)^{\alpha}\right) \mathrm{d} s \pi(\mathrm{d} r)>0 .
$$

We shall give sufficient conditions for $Y$ to be a stationary ID process and also regularly varying of index $\alpha$; see Section 3 .

Extreme-value theory for stable MA processes was derived by Rootzén [31]. We extend Rootzén's results to the much richer class of regularly varying mixed MA processes. Furthermore, we weaken his assumptions on the kernel function. Thus, our model also includes heavy-tailed long-memory processes.

The paper is organized as follows. We start with preliminaries in Section 2, introducing multivariate regular variation (in Section 2.1) and point processes of multivariate regularly varying sequences (in Section 2.2). An investigation of heavy-tailed mixed MA processes follows in Section 3. This includes conditions sufficient for Condition 1.2 to hold, followed by a study of the tail behavior of $Y$ and the tail behavior of $M(h)=\sup _{t \in[0, h]} Y(t)$ for $h>0$. Finally, we introduce supOU processes as examples of heavy-tailed mixed MA processes that can exhibit long-range dependence.

Our main results are presented in Section 4. In Section 4.1, our investigation of the extremal behavior of $Y$ is based on marked point processes on a properly chosen discrete-time skeleton, i.e. one chosen by the jump times of the underlying driving Lévy process in combination with extremes of the kernel function. The marked point processes converge to a marked cluster Poisson random measure. In the neighborhood of such an extreme event, the behavior of the process is solely determined by the kernel function. Finally, in Section 4.2, we obtain the limit distribution of the running maxima of $Y$. The results are applied in particular to supOU processes, in Section 4.3. We conclude with the rather technical proofs of Lemma 2.2, Proposition 3.2, Theorem 4.1, and Theorem 4.2, in Section 5.

Throughout the paper we use the following notation. We write $X \stackrel{\mathrm{D}}{=} Y$ if the distributions of the RVs $X$ and $Y$ coincide. For a vector $\boldsymbol{x} \in \mathbb{R}^{d}$, we denote by $\boldsymbol{x}^{\top}$ the transpose of $\boldsymbol{x}$ and by $|\boldsymbol{x}|=\max \left\{\left|x_{1}\right|, \ldots,\left|x_{d}\right|\right\}$ the maximum norm. For a matrix $\boldsymbol{A} \in \mathbb{R}^{d \times r}$, we denote by $\|\boldsymbol{A}\|$ the operator norm. For a measure $\pi$, we denote by $\operatorname{supp}(\pi)$ the support of $\pi$. Furthermore, $\sum_{k=1}^{0}:=0, \bigvee_{k=1}^{0}:=0$, and $\mathbb{D}(\mathbb{R})$ is the space of càdlàg functions on $\mathbb{R}$ (i.e. those that are continuous from the right and have left limits). Finally, $\varepsilon_{A}$ is the Dirac measure in set $A$.

\section{Preliminaries}

\subsection{Multivariate regular variation}

In Section 4.1, we shall find that the finite-dimensional distributions of $Y$ are multivariate regularly varying. We start with the definition of this notion.

Definition 2.1. (Multivariate regular variation.) A random vector $\boldsymbol{X}=\left(X_{1}, \ldots, X_{d}\right)$ on $\mathbb{R}^{d}$ is said to be regularly varying of index $\alpha, \alpha>0$, if there exists a random vector $\boldsymbol{\Theta}$, taking 
values on the unit $(d-1)$-dimensional sphere $\mathbb{S}^{d-1}=\left\{\boldsymbol{x} \in \mathbb{R}^{d}:|\boldsymbol{x}|=1\right\}$, such that, for every $x>0$,

$$
\frac{\mathrm{P}(|\boldsymbol{X}|>u x, \boldsymbol{X} /|\boldsymbol{X}| \in \cdot)}{\mathrm{P}(|\boldsymbol{X}|>u)} \stackrel{\mathrm{w}}{\rightarrow} x^{-\alpha} \mathrm{P}(\boldsymbol{\Theta} \in \cdot) \quad \text { on } \mathcal{B}\left(\mathbb{S}^{d-1}\right), \quad \text { as } u \rightarrow \infty .
$$

We write $\boldsymbol{X} \in \mathcal{R}_{-\alpha}$.

The distribution of $\boldsymbol{\Theta}$ is referred to as the spectral measure of $\boldsymbol{X}$. It describes in which direction we are likely to find extreme realizations of $\boldsymbol{X}$. An equivalent definition of regular variation is as follows. The vector $\boldsymbol{X}$ is regularly varying if there exist a Radon measure $\sigma(\cdot)$ on $\overline{\mathbb{R}}^{d} \backslash\{\boldsymbol{0}\}$ with $\sigma\left(\overline{\mathbb{R}}^{d} \backslash \mathbb{R}^{d}\right)=0$ and $\sigma(E)>0$ for at least one relatively compact set $E \subseteq \overline{\mathbb{R}}^{d} \backslash\{\mathbf{0}\}$, where $\mathbf{0}=(0, \ldots, 0) \in \mathbb{R}^{d}$, and a sequence of constants $a_{n}$ such that $a_{n}>0, a_{n} \uparrow \infty$, and

$$
n \mathrm{P}\left(a_{n}^{-1} \boldsymbol{X} \in \cdot\right) \stackrel{\mathrm{v}}{\rightarrow} \sigma(\cdot) \quad \text { on } \mathcal{B}\left(\overline{\mathbb{R}}^{d} \backslash\{\mathbf{0}\}\right), \quad \text { as } n \rightarrow \infty .
$$

Then, there exists an $\alpha>0$ such that $\sigma(x B)=x^{-\alpha} \sigma(B)$ for $x>0$ and $B \in \mathscr{B}\left(\overline{\mathbb{R}}^{d} \backslash\{\mathbf{0}\}\right)$. More about multivariate regular variation can be found, e.g. in [4], [25], and [30, Chapter 5, p. 250-306].

The following lemma is a multivariate extension of Breiman's [8] classical result on the regular variation of products and [5, Proposition A.1]. The representation of the spectral measure explicitly given in the lemma follows from straightforward calculations.

Lemma 2.1. Let $\boldsymbol{Z}=\left(Z_{1}, \ldots, Z_{r}\right)$ be a vector of independent $R V s$, regularly varying of index $\alpha$, such that, for $j=1, \ldots, r$, there exists a sequence of constants $a_{n}$ such that $a_{n}>0, a_{n} \uparrow \infty$, and

$$
n \mathrm{P}\left(a_{n}^{-1} Z_{j} \in \cdot\right) \stackrel{\mathrm{v}}{\rightarrow} \sigma_{j}(\cdot) \quad \text { on } \mathcal{B}(\overline{\mathbb{R}} \backslash\{0\}) \text {, as } n \rightarrow \infty,
$$

where

$$
\sigma_{j}(\mathrm{~d} x)=p_{j} \alpha x^{-\alpha-1} 1_{(0, \infty)}(x) \mathrm{d} x+q_{j} \alpha(-x)^{-\alpha-1} 1_{(-\infty, 0)}(x) \mathrm{d} x
$$

with $p_{j}, q_{j} \geq 0$ and $p_{j}+q_{j}>0$. Furthermore, let $\boldsymbol{A}=\left(\boldsymbol{a}_{1}, \ldots, \boldsymbol{a}_{r}\right)$ be a random $d \times r$ matrix, independent of $\boldsymbol{Z}$. If $0<\mathrm{E}\left[\|\boldsymbol{A}\|^{\gamma}\right]<\infty$ for some $\gamma>\alpha$, then $\boldsymbol{Y}=\boldsymbol{A} \boldsymbol{Z}$ is regularly varying of index $\alpha$ and has spectral measure

$$
\mathrm{P}(\boldsymbol{\Theta} \in \cdot)=\frac{\sum_{j=1}^{r}\left(p_{j} \mathrm{E}\left[\left|\boldsymbol{a}_{j}\right|^{\alpha} 1_{\left\{\boldsymbol{a}_{j} /\left|\boldsymbol{a}_{j}\right| \in \cdot\right\}}\right]+q_{j} \mathrm{E}\left[\left|\boldsymbol{a}_{j}\right|^{\alpha} 1_{\left\{-\boldsymbol{a}_{j} /\left|\boldsymbol{a}_{j}\right| \in \cdot\right\}}\right]\right)}{\sum_{j=1}^{r}\left(p_{j}+q_{j}\right) \mathrm{E}\left[\left|\boldsymbol{a}_{j}\right|^{\alpha}\right]} .
$$

For $x>0$, we have

$$
\lim _{n \rightarrow \infty} n \mathrm{P}\left(|\boldsymbol{Y}|>a_{n} x\right)=x^{-\alpha} \sum_{j=1}^{r}\left(p_{j}+q_{j}\right) \mathrm{E}\left[\left|\boldsymbol{a}_{j}\right|^{\alpha}\right],
$$

and

$$
\lim _{n \rightarrow \infty} n \mathrm{P}\left(Y>a_{n} x\right)=x^{-\alpha} \sum_{j=1}^{r}\left(p_{j} \mathrm{E}\left[a_{j}^{+}\right]^{\alpha}+q_{j} \mathrm{E}\left[a_{j}^{-}\right]^{\alpha}\right)
$$

for $d=1$.

Remark 2.1. Let $\boldsymbol{A}$ be a deterministic matrix and let $\rho:=\sum_{j=1}^{r}\left(p_{j}+q_{j}\right) \mathrm{E}\left[\left|\boldsymbol{a}_{j}\right|^{\alpha}\right]$. An interpretation of (2.2) is that the spectral measure $\Theta$ takes the value $\boldsymbol{a}_{j} /\left|\boldsymbol{a}_{j}\right|$ with probability $p_{j}\left|\boldsymbol{a}_{j}\right|^{\alpha} / \rho$ and the value $-\boldsymbol{a}_{j} /\left|\boldsymbol{a}_{j}\right|$ with probability $q_{j}\left|\boldsymbol{a}_{j}\right|^{\alpha} / \rho$. Thus, only in the directions $\boldsymbol{a}_{j} /\left|\boldsymbol{a}_{j}\right|$ and $-\boldsymbol{a}_{j} /\left|\boldsymbol{a}_{j}\right|, j=1, \ldots, r$, are extremes likely to occur. 


\subsection{Point process convergence}

We follow [30] and introduce point processes to describe the extremal behavior of $Y$. In order to achieve distributional stability of a sequence of point processes, it is necessary to allow for an accumulation of infinite mass at $[s, t) \times\{\mathbf{0}\}$. In our setup, this is achieved by defining the state space

$$
S=[0, \infty) \times \overline{\mathbb{R}}^{d} \backslash\{\mathbf{0}\}, \quad d \in \mathbb{N} .
$$

Then $S$ can be metricized as a locally compact, complete, separable Hausdorff space. Compact sets in $S$ are closed sets that are bounded away from 0 . Furthermore, $\mathcal{B}(S)$ denotes the Borel $\sigma$ field on $S$ and $M_{\mathrm{P}}(S)$ the class of point measures on $S$, equipped with the metric $\rho$ that generates the topology of vague convergence. The space $\left(M_{\mathrm{P}}(S), \rho\right)$ is a complete, separable metric space with Borel $\sigma$-field $\mathcal{M}_{\mathrm{P}}(S)$. A point process in $S$ is a random element of $\left(M_{\mathrm{P}}(S), \mathcal{M}_{\mathrm{P}}(S)\right)$, i.e. a measurable map from a probability space $(\Omega, \mathcal{F}, \mathrm{P})$ into $\left(M_{\mathrm{P}}(S), \mathcal{M}_{\mathrm{P}}(S)\right)$. A typical example of a point process in extreme-value theory is a Poisson random measure: given a Radon measure $\vartheta$ on $\mathcal{B}(S)$, a point process $\kappa$ is called a Poisson random measure with intensity measure $\vartheta$, denoted by $\operatorname{PRM}(\vartheta)$, if

(a) $\kappa(A)$ is Poisson distributed with intensity $\vartheta(A)$ for every $A \in \mathcal{B}(S)$, and

(b) for mutually disjoint sets $A_{1}, \ldots, A_{n} \in \mathcal{B}(S), n \in \mathbb{N}$, the RVs $\kappa\left(A_{1}\right), \ldots, \kappa\left(A_{n}\right)$ are independent.

More about point process theory can be found in [10] and [21]. Furthermore, the results of Davis and Hsing [11] on the point process behavior of a stationary sequence of regularly varying RVs under weak dependence are of vital importance for our study. These results were generalized in [12] to multidimensional regularly varying stationary processes, which are used in Section 4.1 .

The following lemma shows that the addition of a sequence of 'small' random vectors to a sequence of multivariate regularly varying random vectors has no influence on the point process behavior. By 'small' we mean that the tail of the norm value of the random vector decreases faster than the tail of the norm value of the multivariate regularly varying random vectors. Let $N$ be a point process with jump times $\left\{\Gamma_{k}\right\}_{k \in \mathbb{N}}$ labeled such that $0<\Gamma_{1}<\Gamma_{2}<\cdots<\infty$. If the interarrival times $\left\{\Gamma_{k+1}-\Gamma_{k}\right\}_{k \in \mathbb{N}}$ are independent and identically distributed (i.i.d.), then the counting process $N$ is said to be a renewal process with intensity $\mu:=\mathrm{E}\left[\Gamma_{2}-\Gamma_{1}\right]$. We write $\lfloor\cdot\rfloor$ for the integer-part function.

Lemma 2.2. Let $\boldsymbol{Z}=\left\{\boldsymbol{Z}_{k}\right\}_{k \in \mathbb{N}}$ and $\boldsymbol{\Psi}=\left\{\boldsymbol{\Psi}_{k}\right\}_{k \in \mathbb{N}}$ be sequences of random vectors in $\mathbb{R}^{d}$. Furthermore, let $\left\{\Gamma_{k}\right\}_{k \in \mathbb{N}}$ be the jump times of a renewal process $N$ with intensity $\mu>0$, let $h \in \mathbb{R}$ be arbitrary, and let

$$
s_{k} \in\left[\Gamma_{k-1}+h, \Gamma_{k+1}+h\right) \quad \text { for } k \in \mathbb{N},
$$

setting $\Gamma_{0}:=0$. Consider a sequence of constants $a_{n}$ with $a_{n}>0$ and $a_{n} \uparrow \infty$, and define the point processes

$$
\begin{aligned}
\tilde{\kappa}_{n} & =\sum_{k=1}^{\infty} \varepsilon_{\left(k / n, \boldsymbol{Z}_{k} / a_{n}\right)}, \quad n \in \mathbb{N}, \\
\kappa_{T} & =\sum_{k=1}^{\infty} \varepsilon_{\left(s_{k} \mu / T,\left(\boldsymbol{Z}_{k}+\boldsymbol{\Psi}_{k}\right) / a_{\lfloor T\rfloor}\right)}, \quad T>0,
\end{aligned}
$$


in $M_{\mathrm{P}}(S)$. Suppose that there exists a point process $\kappa$ in $M_{\mathrm{P}}(S)$, with $\kappa([s, t) \times\{\boldsymbol{x}\})=0$ a.s. for $\boldsymbol{x} \in \overline{\mathbb{R}}^{d} \backslash\{\mathbf{0}\}, t>s \geq 0$, such that

$$
\tilde{\kappa}_{n} \stackrel{\mathrm{W}}{\rightarrow} \kappa \text { as } n \rightarrow \infty .
$$

Furthermore, assume that, for every $\epsilon, t>0$,

$$
\sum_{k=1}^{\lfloor n t\rfloor} \mathrm{P}\left(\left|\boldsymbol{\Psi}_{k}\right|>a_{n} \epsilon\right) \rightarrow 0, \quad n \rightarrow \infty .
$$

Moreover, we suppose that there exists an $R V W$ such that

$$
\begin{aligned}
& \mathrm{P}\left(\left|\boldsymbol{Z}_{k}+\boldsymbol{\Psi}_{k}\right|>x\right) \leq \mathrm{P}(W>x) \text { for } x>0 \text {, } \\
& \mathrm{P}\left(W>a_{n} x\right)=O(1 / n) \quad \text { as } n \rightarrow \infty .
\end{aligned}
$$

Let $I=[s, t) \times \prod_{i=1}^{d}\left(c_{i}, d_{i}\right] \subseteq S$ be bounded away from $\mathbf{0}$. Then

$$
\lim _{T \rightarrow \infty} \mathrm{P}\left(\kappa_{T}(I) \neq \tilde{\kappa}_{\lfloor T\rfloor}(I)\right)=0
$$

and $\kappa_{T} \stackrel{\mathrm{w}}{\rightarrow} \kappa$ as $T \rightarrow \infty$.

Note that $\left\{\boldsymbol{Z}_{k}\right\}_{k \in \mathbb{N}}$ and $\left\{\boldsymbol{Z}_{k}+\boldsymbol{\Psi}_{k}\right\}_{k \in \mathbb{N}}$ need not be stationary sequences. To provide some intuition for random vectors $\left\{\boldsymbol{\Psi}_{k}\right\}_{k \in \mathbb{N}}$ satisfying (2.3), we give some examples.

Example 2.1. (a) Assume that there exists an RV $\psi$ such that, for some $x_{0} \geq 0$ and any $\epsilon>0$ and $k \in \mathbb{N}$,

$$
\begin{array}{ll}
\mathrm{P}\left(\left|\boldsymbol{\Psi}_{k}\right|>x\right) \leq \mathrm{P}(\psi>x) & \text { for } x \geq x_{0}, \\
\mathrm{P}\left(\psi>a_{n} \epsilon\right)=o(1 / n) & \text { as } n \rightarrow \infty
\end{array}
$$

Then (2.3) is satisfied.

(b) Let $\left\{\tilde{Z}_{k}\right\}_{k \in \mathbb{N}}$ be a sequence of identically distributed RVs that are regularly varying of index $\alpha$ in the sense of (2.1) with the $a_{n}$ given in Lemma 2.2. Suppose that $\left\{\tilde{Z}_{k}\right\}_{k \in \mathbb{N}}$ is independent of the sequence of random vectors $\left\{\tilde{\boldsymbol{\Psi}}_{k}\right\}_{k \in \mathbb{N}}$ in $\mathbb{R}^{d}$, which have support on $\left[-f^{+}, f^{+}\right]^{d}$. Define $\boldsymbol{\Psi}_{k}:=\tilde{\boldsymbol{\Psi}}_{k} \tilde{Z}_{k}$ and assume that there exists a $\delta, 0<\delta<\alpha$, such that

$$
\sum_{k=-\infty}^{\infty} \mathrm{E}\left[\left|\tilde{\boldsymbol{\Psi}}_{k}\right|^{\delta}\right]<\infty
$$

Denote by $F_{k}$ the DF of $\tilde{\boldsymbol{\Psi}}_{k}$. By Potter's theorem (see [6, Theorem 1.5.6, p. 25]) there exist an $n_{0} \in \mathbb{N}$ and a $K>1$ such that, for $k \in \mathbb{N}$ and $n \geq n_{0}$,

$$
\begin{aligned}
\mathrm{P}\left(\left|\tilde{\boldsymbol{\Psi}}_{k} \tilde{Z}_{k}\right|>a_{n} \epsilon\right) & =\int_{\overline{\mathbb{R}}^{d} \backslash\{\boldsymbol{0}\}} \mathrm{P}\left(f^{+}\left|\tilde{Z}_{k}\right|>a_{n} \epsilon f^{+} /|\boldsymbol{t}|\right) F_{k}(\mathrm{~d} \boldsymbol{t}) \\
& \leq K \mathrm{P}\left(f^{+}\left|\tilde{Z}_{1}\right|>a_{n} \epsilon\right) \mathrm{E}\left[\left|\tilde{\boldsymbol{\Psi}}_{k}\right|^{\delta}\right] .
\end{aligned}
$$

From this and the fact that $a_{n} \rightarrow \infty$ as $n \rightarrow \infty$, we obtain

$$
\lim _{n \rightarrow \infty} \sum_{k=1}^{\lfloor n t\rfloor} \mathrm{P}\left(\left|\boldsymbol{\Psi}_{k}\right|>a_{n} \epsilon\right) \leq K \lim _{n \rightarrow \infty} \mathrm{P}\left(f^{+}\left|\tilde{Z}_{1}\right|>a_{n} \epsilon\right) \sum_{k=1}^{\infty} \mathrm{E}\left[\left|\tilde{\Psi}_{k}\right|^{\delta}\right]=0 .
$$

Thus, $\left\{\boldsymbol{\Psi}_{k}\right\}_{k \in \mathbb{N}}$ satisfies (2.3). 


\section{Stationarity and tail behavior of $Y$}

This paper is concerned with extremes of regularly varying mixed MA processes $Y$, as given in (1.1), which means that the underlying driving Lévy process satisfies Condition 1.1. Under certain conditions, $Y$ is well defined as a limit in probability of integrals of step functions approximating $f$. This has been shown by Rajput and Rosiński [29, Theorem 2.7] (see also [22]). They gave necessary and sufficient conditions formulated in terms of the kernel function $f$ and the generating quadruple $\left(m, \sigma^{2}, v, \pi\right)$ of the IDISRM $\Lambda$. Under these conditions, $Y$ is ID and, owing to the structure of mixed MA processes, stationary. The following proposition gives conditions sufficient to ensure that these former conditions are satisfied. For details of the proof, we refer the reader to [15, Proposition 2.2.3].

Proposition 3.1. (Existence.) Let $\Lambda$ be an IDISRM with generating quadruple $\left(m, \sigma^{2}, v, \pi\right)$, let the underlying driving Lévy process $L$, as defined in (1.2), satisfy Condition 1.1, and let $f$ be bounded. Then $Y$, given by (1.1), is well defined, ID, and stationary if one of the following conditions is satisfied:

(a) $L(1)$ is $\alpha$-stable, $\alpha \in(0,1) \cup(1,2)$, and $f \in \mathbb{L}^{\alpha}(\pi)$;

(b) $f \in \mathbb{L}^{\delta}(\pi)$ for some $\delta$ with $\delta<\alpha$ and $\delta \leq 1$;

(c) $\mathrm{E}[L(1)]=0, \alpha>1$, and $f \in \mathbb{L}^{\delta}(\pi)$ for some $\delta$ with $\delta<\alpha$ and $\delta \leq 2$.

Remark 3.1. (i) For a Lévy-driven MA process as given in (1.3), Proposition 3.1 provides sufficient conditions for $Y$ to be stationary and the marginal distribution to be ID. We can thus replace $\mathbb{L}^{\delta}(\pi)$ by $\mathbb{L}^{\delta}$. Typical examples of functions in $\mathbb{L}^{\delta}$ are bounded functions $f$ with $f(t) \sim K_{1} t^{-\delta+\epsilon}$ and $f(-t) \sim K_{2} t^{-\delta+\epsilon}$ as $t \rightarrow \infty$, for some $\epsilon \in(0, \delta)$ and $K_{1}, K_{2} \in \mathbb{R}$. OU processes, continuous-time autoregressive MA processes, and stochastic delay equations, which have exponentially decreasing kernel functions, satisfy this condition, as do fractionally integrated continuous-time autoregressive MA processes.

(ii) Let $Y$ be a stationary mixed MA process given by (1.1) with kernel function $f$ and generating quadruple $\left(m, \sigma^{2}, v, \pi\right)$ of $\Lambda$. Then $f \in \mathbb{L}^{\alpha+\epsilon}(\pi)$ for some $\epsilon>0$.

Proposition 3.2. (Tail behavior.) Let $Y$ be a mixed MA process, given by (1.1), satisfying Condition 1.2, and let $x>0$. Then, for $t \in \mathbb{R}$,

$$
\lim _{n \rightarrow \infty} n \mathrm{P}\left(Y(t)>a_{n} x\right)=x^{-\alpha} \int_{\mathbb{R}_{+}} \int_{\mathbb{R}}\left(p\left(f^{+}(r, s)\right)^{\alpha}+q\left(f^{-}(r, s)\right)^{\alpha}\right) \mathrm{d} s \pi(\mathrm{d} r) .
$$

Moreover, for $t_{i} \in \mathbb{R}, i=1, \ldots, k, k \in \mathbb{N}$,

$$
\lim _{n \rightarrow \infty} n \mathrm{P}\left(\max _{i=1, \ldots, k}\left|Y\left(t_{i}\right)\right|>a_{n} x\right)=x^{-\alpha} \int_{\mathbb{R}_{+}} \int_{\mathbb{R}} \max _{i=1, \ldots, k}\left|f\left(r, t_{i}-s\right)\right|^{\alpha} \mathrm{d} s \pi(\mathrm{d} r) .
$$

Furthermore, let $f_{h}(r, s)=\sup _{t \in[0, h]}|f(r, t+s)| \in \mathbb{L}^{\alpha-\epsilon}(\pi)$ for some $\epsilon, 0<\epsilon<\alpha$, and let $M(h)=\sup _{t \in[0, h]} Y(t)$. Then

$$
\begin{aligned}
\lim _{n \rightarrow \infty} & n \mathrm{P}\left(M(h)>a_{n} x\right) \\
= & x^{-\alpha} \int_{\mathbb{R}_{+}} \int_{\mathbb{R}}\left(p \sup _{t \in[0, h]}\left(f^{+}(r, t+s)\right)^{\alpha}+q \sup _{t \in[0, h]}\left(f^{-}(r, t+s)\right)^{\alpha}\right) \mathrm{d} s \pi(\mathrm{d} r) .
\end{aligned}
$$

From (3.1) we see that $Y(t)$ is again regularly varying in the sense of (2.1). 
Example 3.1. (supOU process.) We consider the mixed MA process given in (1.1), where the kernel function is $f(r, s)=1_{[0, \infty)}(s) \mathrm{e}^{-r s}$ for $r \in \mathbb{R}_{+}$and $s \in \mathbb{R}$. Then

$$
Y(t)=\int_{\mathbb{R}_{+} \times \mathbb{R}} 1_{[0, \infty)}(t-s) \mathrm{e}^{-r(t-s)} \mathrm{d} \Lambda(r, s), \quad t \in \mathbb{R},
$$

is called a supOU process. An important special case of (3.4) is the OU process, for which $\pi$ has support only at some $\lambda>0$, i.e. $\pi(\{\lambda\})=1$.

For a general probability measure $\pi$ and for some $\delta>0$, we have $f \in \mathbb{L}^{\delta}(\pi)$ if and only if

$$
\int_{\mathbb{R}_{+}} \int_{\mathbb{R}_{+}} \mathrm{e}^{-r s \delta} \mathrm{d} s \pi(\mathrm{d} r)=\delta^{-1} \int_{\mathbb{R}_{+}} r^{-1} \pi(\mathrm{d} r)<\infty .
$$

We assume in the following that $\lambda^{-1}:=\int_{\mathbb{R}_{+}} r^{-1} \pi(\mathrm{d} r)<\infty$. Hence, $f \in \mathbb{L}^{\delta}(\pi)$ for every $\delta>0$. The conditions necessary and sufficient for supOU processes to exist and be ID (see [29, Theorem 2.7]) reduce to the conditions necessary and sufficient for a simple OU process with parameter $\lambda$ to exist. Then, by [34, Theorem 17.5, p. 108-109], the supOU process exists and is ID if and only if $\int_{|x|>2} \log |x| v(\mathrm{~d} x)<\infty$. We obtain the generating triplet

$$
\begin{array}{ccrl}
m_{Y}=\frac{1}{\lambda}\left[m+\int_{|y|>1} \frac{y}{|y|} v(\mathrm{~d} y)\right], & \sigma_{Y}^{2}=\frac{\sigma^{2}}{2 \lambda}, \\
v_{Y}[x, \infty)=\frac{1}{\lambda} \int_{x}^{\infty} \frac{v[y, \infty)}{y} \mathrm{~d} y, & x>0 .
\end{array}
$$

Note that the finite-dimensional distributions of $Y$ are those of an OU process with parameter $\lambda$, whose driving Lévy process has characteristic triplet $\left(m, \sigma^{2}, v\right)$, i.e. the marginal distribution of $Y$ is self-decomposable. Furthermore, for any regularly varying Lévy process satisfying Condition 1.1, $Y$ is a stationary ID process and

$$
\lim _{n \rightarrow \infty} n \mathrm{P}\left(Y(t)>a_{n} x\right)=\frac{1}{\lambda \alpha} x^{-\alpha} \quad \text { for } t \in \mathbb{R} .
$$

Define the probability measure $\bar{\pi}(\mathrm{d} r)=\lambda r^{-1} \pi(\mathrm{d} r)$ and the IDISRM $\bar{\Lambda}$ with generating quadruple $\left(m / \lambda, \sigma^{2} / \lambda, v / \lambda, \bar{\pi}\right)$. Then the finite-dimensional distributions of the stochastic process

$$
X(t)=\int_{-\infty}^{\infty} \mathrm{e}^{-r t} \int_{-\infty}^{r t} \mathrm{e}^{s} \mathrm{~d} \bar{\Lambda}(r, s), \quad t \in \mathbb{R},
$$

coincide with those of $Y$, i.e. $X \stackrel{\text { D }}{=} Y$ (see [2, Theorem 3.1]). Since

$$
\mathrm{d} X(t)=\int_{\mathbb{R}_{+}}\{-r X(t, \mathrm{~d} r) \mathrm{d} t+\mathrm{d} \bar{\Lambda}(t, r)\}, \quad t \in \mathbb{R},
$$

with $X(t, B)=\int_{B} \mathrm{e}^{r t} \int_{-\infty}^{r t} \mathrm{e}^{s} \mathrm{~d} \bar{\Lambda}(r, s)$ for $t \in \mathbb{R}$ and $B \in \mathscr{B}(\mathbb{R})$, the process $X$ and, hence, the process $Y$ are called supOU processes.

For a proper choice of $\pi$, the correlation function $\rho(h)=\lambda \int_{0}^{\infty} r^{-1} \mathrm{e}^{-h r} \pi(\mathrm{d} r), h \in \mathbb{R}$, can be used to model long-memory processes. For example, if $\pi$ is gamma distributed with density $\pi(\mathrm{d} r)=\Gamma(2 H+1)^{-1} r^{2 H} \mathrm{e}^{-r} \mathrm{~d} r$ for $r>0$ and $H>0$, then

$$
\rho(h)=\frac{1}{\Gamma(2 H)} \int_{0}^{\infty} r^{2 H-1} \mathrm{e}^{-r(h+1)} \mathrm{d} r=\frac{1}{(h+1)^{2 H}}, \quad h \in \mathbb{R} .
$$

More details about supOU models and their relevance in applications to financial data can be found in [3]. 


\section{Extremal behavior}

\subsection{The point process of a discrete-time skeleton}

In this section, we study the extremal behavior of a regularly varying mixed MA process. To this end, we use a discrete-time skeleton for a process $Y$, as given in (1.1), satisfying Condition 1.2. This means that we investigate the extremal behavior of a discrete-time sequence $\left\{Y\left(t_{n}\right)\right\}_{n \in \mathbb{N}}$, where the discrete-time random sequence $\left\{t_{n}\right\}_{n \in \mathbb{N}}$ is chosen properly by the jump times of the underlying driving Lévy process $L$, as given in (1.2), and the extremes of the kernel function. We shall show that the extremes of $\left\{Y\left(t_{n}\right)\right\}_{n \in \mathbb{N}}$ coincide with extremes of $Y$ at high levels.

We therefore decompose $\Lambda$ into two independent IDISRMs according to the jump sizes of the underlying driving Lévy process $L$, which are represented by $\nu$. We define

$$
\Lambda=\Lambda_{1}+\Lambda_{2}, \quad \text { with } \quad \Lambda_{1}(A)=\int_{\mathbb{R}} x \mathrm{~d} \tilde{N}_{1}(A, x) \quad \text { for } A \in \mathcal{A},
$$

where $\tilde{N}_{1}$ is a Poisson random measure $\operatorname{PRM}(\vartheta)$ with intensity

$$
\vartheta(\mathrm{d} r \times \mathrm{d} t \times \mathrm{d} x)=\pi(\mathrm{d} r) \times \mathrm{d} t \times v_{1}(\mathrm{~d} x),
$$

and $v_{1}$ is the Lévy measure:

$$
v_{1}(A)=v(A \cap(1, \infty))+v(A \cap(-\infty,-1)), \quad A \in \mathcal{B}(\mathbb{R}) .
$$

The generating quadruple of $\Lambda_{1}$ is $\left(0,0, \nu_{1}, \pi\right)$, and $\Lambda_{1}$ is called a compound Poisson random measure. The IDISRM $\Lambda_{2}$ has the generating quadruple $\left(m, \sigma^{2}, \nu_{2}, \pi\right)$ with Lévy measure $v_{2}=v-v_{1}$, i.e. it has finite support. We refer the reader to [28] for the Lévy-Itô decomposition of IDISRMs. The underlying driving Lévy process of $\Lambda_{1}$ has generating triplet $\left(0,0, v_{1}\right)$ and jumps of modulus larger than one, while the underlying driving Lévy process of $\Lambda_{2}$ has generating triplet $\left(m, \sigma^{2}, v_{2}\right)$ and jumps of modulus smaller than one. Furthermore, $\tilde{N}_{1}$ has the representation

$$
\tilde{N}_{1}=\sum_{k=-\infty}^{\infty} \varepsilon_{\left(R_{k}, \Gamma_{k}, Z_{k}\right)}
$$

where $-\infty<\cdots<\Gamma_{-1}<\Gamma_{0} \leq 0<\Gamma_{1}<\cdots<\infty$ are the jump times of a Poisson process $N=\{N(t)\}_{t \in \mathbb{R}}$ with intensity $\mu=v_{1}(\mathbb{R})>0, Z=\left\{Z_{k}\right\}_{k \in \mathbb{Z}}$ is an i.i.d. sequence with DF $\mathrm{P}\left(Z_{1} \leq x\right)=v_{1}(-\infty, x] / \mu$ for $x \in \mathbb{R}$, and $R=\left\{R_{k}\right\}_{k \in \mathbb{Z}}$ is an i.i.d. sequence with DF $\pi$. The processes $N, Z$, and $R$ are independent. It is also possible to choose a different decomposition in (4.1) by using a Poisson random measure and an IDISRM whose underlying driving Lévy process has bounded support in a neighborhood of the origin.

This decomposition of $\Lambda$ induces the decomposition $Y=Y_{1}+Y_{2}$, where, for $i=1,2$,

$$
Y_{i}(t)=\int_{\mathbb{R}_{+} \times \mathbb{R}} f(r, t-s) \mathrm{d} \Lambda_{i}(r, s), \quad t \in \mathbb{R},
$$

are independent mixed MA processes. Without loss of generality, we assume that $Y_{1}$ and $Y_{2}$ are stationary, measureable, and separable ID processes. We shall see that the extremal behavior of a mixed MA process $Y$ satisfying Condition 1.2 is completely determined by the extremes of the mixed Poisson shot noise process $Y_{1}$ with representation

$$
Y_{1}(t)=\sum_{k=-\infty}^{\infty} f\left(R_{k}, t-\Gamma_{k}\right) Z_{k}, \quad t \in \mathbb{R}
$$


We first give a short motivation for the choice of the discrete-time random sequence $\left\{t_{n}\right\}_{n \in \mathbb{N}}$. Suppose that there exists an $\eta^{(1)} \in \mathbb{R}$ with $f\left(r, \eta^{(1)}\right)=f^{+}$for every $r \in \operatorname{supp}(\pi)$. The mixed Poisson shot noise process $Y_{1}$ can then be written

$$
Y_{1}\left(\Gamma_{k}+t\right)=f\left(R_{k}, t\right) Z_{k}+\sum_{\substack{j=-\infty \\ j \neq k}}^{\infty} f\left(R_{j}, t+\Gamma_{k}-\Gamma_{j}\right) Z_{j}, \quad k \in \mathbb{N}, t \in \mathbb{R}
$$

In the case in which the $\left\{Z_{k}\right\}_{k \in \mathbb{Z}}$ are regularly varying, one of the $Z_{k}$ is likely to be large in comparison to $\left\{Z_{j}\right\}_{j \in \mathbb{Z} \backslash\{k\}}$, meaning that $Y_{1}\left(\Gamma_{k}+t\right)$ behaves roughly like $f\left(R_{k}, t\right) Z_{k}$. The process $\left\{f\left(R_{k}, t\right) Z_{k}\right\}_{t \geq 0}$ achieves a maximum at $t=\eta^{(1)}$. Similar results hold for large negative jumps and a minimum, $\eta^{(2)}$, of the kernel function with $f\left(r, \eta^{(2)}\right)=-f^{-}$for every $r \in \operatorname{supp}(\pi)$. This suggests that $Y_{1}\left(t_{n}\right)$, with

$$
t_{n} \in\left\{\Gamma_{k}+\eta^{(1)}, k \in \mathbb{N}\right\} \cup\left\{\Gamma_{k}+\eta^{(2)}, k \in \mathbb{N}\right\},
$$

is a local extreme value of $Y_{1}$ if the absolute value of the jump of the underlying driving Lévy process is large.

Throughout the rest of the paper, we use the following assumptions and notation. Let $t_{1}, \ldots, t_{d-1} \in \mathbb{R}, d \in \mathbb{N}$, be fixed and define $\eta^{(1)}$ as above. Then, for $t \in \mathbb{R}$, we define

$$
\begin{aligned}
f(r, t) & :=\left(f\left(r, t+t_{1}\right), \ldots, f\left(r, t+t_{d-1}\right), f\left(r, t+\eta^{(1)}\right)\right), \\
\boldsymbol{Y}(t) & :=\left(Y\left(t+t_{1}\right), \ldots, Y\left(t+t_{d-1}\right), Y\left(t+\eta^{(1)}\right)\right) .
\end{aligned}
$$

The extremal behavior of $Y$ is described by the multivariate point processes

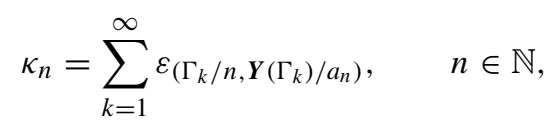

in $M_{\mathrm{P}}(S)$. Such point processes can be interpreted as marked point processes (see [10, Section 6.4, p. 194-210]). Let $Y_{k, i}=Y\left(\Gamma_{k}+t_{i}\right), i \in\{1, \ldots, d\}$, be the $i$ th coordinate of $Y\left(\Gamma_{k}\right)$, where $t_{d}:=\eta_{1}$. In a marked point process, we consider the point process behavior of $\sum_{k=1}^{\infty} \varepsilon_{\left(\Gamma_{k} / n, Y_{k, i} / a_{n}\right)}$ for some fixed $i \in\{1, \ldots, d\}$, and the remaining coordinates of $\boldsymbol{Y}\left(\Gamma_{k}\right)$ describe the behavior of the process when an excess of $Y_{k, i}$ over a high threshold occurs. In our setting, $\left(Y\left(\Gamma_{k}+t_{1}\right), \ldots, Y\left(\Gamma_{k}+t_{d-1}\right)\right) / a_{n}$ are the marks, which describe the sample path behavior of the continuous-time process $Y$ if $Y\left(\Gamma_{k}+\eta^{(1)}\right)$ exceeds a high level. They characterize clearly the locations of extremes at high levels.

We work with the sequence $T=\left\{T_{k}\right\}_{k \in \mathbb{Z}}$, where, for $k \in \mathbb{N}_{0}$,

$$
T_{k}:=\Gamma_{k+1}-\Gamma_{1} \quad \text { and } \quad T_{-k}:=\Gamma_{-k}-\Gamma_{0} .
$$

Hence, $\left\{T_{k}-T_{k-1}\right\}_{k \in \mathbb{Z} \backslash\{0\}}$ is an i.i.d. sequence with $T_{k}-T_{k-1} \stackrel{\mathrm{D}}{=} \Gamma_{1}$ and $T_{0}=0$.

Theorem 4.1. Let $Y$ be a mixed MA process, as given in (1.1), such that Condition 1.2 is satisfied and the kernel function $f$ satisfies $f\left(r, \eta^{(1)}\right)=f^{+} \geq f^{-}$for every $r \in \operatorname{supp}(\pi)$. Let $\sum_{k=1}^{\infty} \varepsilon_{\left(s_{k}, P_{k}\right)}$ be a $\operatorname{PRM}(\vartheta)$ with $\vartheta(\mathrm{d} t \times \mathrm{d} x)=\mathrm{d} t \times \alpha x^{-\alpha-1} 1_{(0, \infty)}(x) \mathrm{d} x$. Suppose that $T^{(k)}, k \in \mathbb{N}$, are i.i.d. with $T^{(k)}=\left\{T_{k, j}\right\}_{j \in \mathbb{Z}} \stackrel{\mathrm{D}}{=} T$, where $T$ is the sequence defined by (4.3), and $R=\left\{R_{k}\right\}_{k \in \mathbb{N}}$ is an i.i.d. sequence with $D F \pi$. Let $\chi=\left\{\chi_{k}\right\}_{k \in \mathbb{N}}$ be an i.i.d. sequence 
with $\mathrm{P}\left(\chi_{k}=1\right)=p$ and $\mathrm{P}\left(\chi_{k}=-1\right)=q$. Furthermore, suppose that the random objects $\sum_{k=1}^{\infty} \varepsilon_{\left(s_{k}, P_{k}\right)},\left\{T^{(k)}\right\}_{k \in \mathbb{N}}, R$, and $\chi$ are independent. Then, as $T \rightarrow \infty$,

$$
\sum_{k=1}^{\infty} \varepsilon_{\left(\Gamma_{k} / T, \boldsymbol{Y}\left(\Gamma_{k}\right) / a_{\lfloor T\rfloor}\right)} \stackrel{\mathrm{w}}{\rightarrow} \sum_{k=1}^{\infty} \sum_{j=-\infty}^{\infty} \varepsilon_{\left(s_{k}, f\left(R_{k}, T_{k, j}\right) \chi_{k} P_{k}\right)}=: \kappa \quad \text { in } M_{\mathrm{P}}(S) .
$$

In particular, for $t$ with $f(r, t) \neq 0$ for $r \in \operatorname{supp}(\pi)$, as $T \rightarrow \infty$ we have

$$
\sum_{k=1}^{\infty} \varepsilon_{\left(\Gamma_{k} / T, \boldsymbol{Y}\left(\Gamma_{k}+t\right) / a_{\lfloor T\rfloor}\right)} \stackrel{\mathrm{w}}{\rightarrow} \sum_{k=1}^{\infty} \sum_{j=-\infty}^{\infty} \varepsilon_{\left(s_{k}, \boldsymbol{f}\left(R_{k}, T_{k, j}+t\right) \chi_{k} P_{k}\right)} \quad \text { in } M_{\mathrm{P}}([0, \infty) \times \overline{\mathbb{R}} \backslash\{0\}) .
$$

The assumption that $f\left(r, \eta^{(1)}\right)=f^{+} \geq f^{-}$for every $r \in \operatorname{supp}(\pi)$ can be replaced by the assumption that $f\left(r, \eta^{(2)}\right)=-f^{-} \leq-f^{+}$for every $r \in \operatorname{supp}(\pi)$.

Remark 4.1. (a) The properly chosen discrete-time points at which exceedances of the underlying driving Lévy process occur in combination with extremes of the kernel function result in exceedances of the mixed MA process. These exceedances are propagated in time by the kernel function and result in clusters of exceedances in the limiting process. Furthermore, they also reflect local extremes of the process at high levels.

(b) Regularly varying DFs are a subclass of subexponential DFs. Subexponential models are typically applied in situations in which values are likely to occur that are extremely large in comparison to the mean size of the data. Regularly varying DFs agree with subexponential DFs in the maximum domain of attraction of the Fréchet distribution. Extremes of subexponential Lévy-driven MA processes, which are in the maximum domain of attraction of the Gumbel distribution, have been studied in [16]. In both classes of subexponential distribution, the large jumps of the Lévy process affect the extremal behavior, which can be modeled by a properly chosen discrete-time skeleton. However, in contrast to the Fréchet case as described in part (a), in the Gumbel case exceedances over high thresholds collapse into single points, which are described by the extremes of the kernel function, meaning that the marked point processes converge to a cluster Poisson random measure with constant cluster sizes.

(c) Theorem 4.1 contains information about the local minima of $Y$, since the point process converges in $M_{\mathrm{P}}\left([0, \infty) \times \overline{\mathbb{R}}^{d} \backslash\{\boldsymbol{0}\}\right)$. The interpretation of small minima is analogous to that of large maxima. They occur in clusters and are caused by large jumps of the underlying driving Lévy process.

(d) It should be possible to extend Theorem 4.1 to an infinite-dimensional setting, where we use as marks the stochastic processes $\left\{Y\left(\Gamma_{k}+t\right)\right\}_{t \in[0, m]}$ in $\mathbb{D}[0, m], m>0$, instead of multidimensional random vectors $\boldsymbol{Y}\left(\Gamma_{k}\right) \in \mathbb{R}^{d}, k \in \mathbb{N}$. The formulation of such results requires the definition of regular variation of stochastic processes with a.s. càdlàg sample paths given in [20]. Moreover, since $\mathbb{D}$ is not locally compact, a special definition of convergence (convergence on bounded Borel sets), given in [10, Section A.2.6, pp. 402-406], is needed.

Corollary 4.1. (Point process of exceedances.) Let $Y$ be as given in Theorem 4.1, with $f^{+} \leq 1$. Suppose that $\tilde{s}_{k}, k \in \mathbb{N}$, are the jump times of a Poisson process with intensity $x^{-\alpha}, x>0$, independent of the i.i.d. sequence $\left\{\zeta_{k}\right\}_{k \in \mathbb{Z}}$ with $D F$

$$
\pi_{k}=\mathrm{P}\left(\zeta_{1}=k\right)=p\left(\mathrm{E}\left[f_{k}^{(1) \alpha}\right]-\mathrm{E}\left[f_{k+1}^{(1) \alpha}\right]\right)+(1-p)\left(\mathrm{E}\left[f_{k}^{(2) \alpha}\right]-\mathrm{E}\left[f_{k+1}^{(2) \alpha}\right]\right), \quad k \in \mathbb{N},
$$


where $f_{1}^{(1)}>f_{2}^{(1)}>\cdots$ are the order statistics of $\left\{f^{+}\left(R_{1}, T_{j}+t\right)\right\}_{j \in \mathbb{Z}}$ and $f_{1}^{(2)}>f_{2}^{(2)}>\cdots$

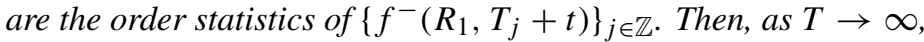

$$
\sum_{k=1}^{\infty} \varepsilon_{\left(\Gamma_{k} / T, Y\left(\Gamma_{k}+t\right) / a_{\lfloor T\rfloor}\right)}(\cdot \times(x, \infty)) \stackrel{\mathrm{w}}{\rightarrow} \sum_{k=1}^{\infty} \zeta_{k} \varepsilon_{\tilde{s}_{k}} .
$$

In the case of a positive shot noise process with nonincreasing kernel function and $t=\eta^{(1)}$, this result gives the cluster intensities among local extremes of the process.

\subsection{Normalizing constants of running maxima}

Using the results of the previous section, we now calculate the normalizing constants of the running maxima. Lebedev [24] calculated the limit distribution of the running maxima of subexponential, positive shot noise processes, restricting his attention to nondecreasing kernel functions with unbounded support. In our result, the assumption of a positive process with nonincreasing kernel function is not necessary.

Theorem 4.2. Let $Y$ be a mixed MA process, as given in (1.1), that has a.s. càdlàg sample paths and satisfies Condition 1.2 and one of the following conditions.

(a) Let $f$, the kernel function of $Y$, be positive. Assume that there exists a measurable function $\tilde{f}: \mathbb{R}_{+} \times \mathbb{R} \rightarrow \mathbb{R}_{+}$such that $f(r, s) \leq \tilde{f}(r, s)$ for $(r, s) \in \mathbb{R}_{+} \times \mathbb{R}, \tilde{f}(r, \cdot)$ is nonincreasing on $\left[\eta^{(1)}, \infty\right)$, and $\tilde{f}\left(r, \eta^{(1)}\right)=f^{+}$for every $r \in \operatorname{supp}(\pi)$. Furthermore, let the support of $\tilde{f}$ be contained in $\mathbb{R}_{+} \times\left[\eta^{(1)}, \infty\right)$ and let $\tilde{f} \in \mathbb{L}^{\delta}(\pi)$ for some $\delta<$ $\min \{1, \alpha\}$.

(b) Let $Y$ be a Lévy-driven MA process with $\int_{-\infty}^{\infty} \sup _{0 \leq s \leq 1}|f(s+t)|^{\delta} \mathrm{d} t<\infty$ for some $\delta<\min \{1, \alpha\}$.

Define $M(T)=\sup _{t \in[0, T]} Y(t)$ for $T>0$. Then

$$
\lim _{T \rightarrow \infty} \mathrm{P}\left(a_{\lfloor T\rfloor}^{-1} M(T) \leq x\right)=\exp \left(-x^{-\alpha}\left[p\left(f^{+}\right)^{\alpha}+q\left(f^{-}\right)^{\alpha}\right]\right) \quad \text { for } x>0 .
$$

Notice that, by the integrability assumption on $f$, this result rules out MA processes that exhibit long-range dependence; only mixed MA processes with long-range dependence are included. In this case, the long-range dependence is caused by the distribution of $\pi$ and not by the asymptotic behavior of the kernel function for fixed $r$.

Theorem 4.2 requires that $p f^{+}+q f^{-}>0$, by Condition 1.2 (otherwise the limit in (4.4) is 1). More about the extremal behavior of totally skewed $\alpha$-stable MA processes, which satisfy $p f^{+}+q f^{-}=0$ (meaning that the right tail is not regularly varying), can be found in [1].

Remark 4.2. (a) The results of this paper can be extended to mixed MA processes driven by a multivariate IDISRM $\Lambda$ in $\mathbb{R}_{+}^{m} \times \mathbb{R}$ whose stationary distribution has the cumulant generating function $\psi_{A}(u)=\lambda(A) \psi(u)$. Here, $\psi$ is the cumulant generating function of a Lévy process,

$$
\lambda(\mathrm{d} \omega)=\pi_{1}\left(\mathrm{~d} r_{1}\right) \times \cdots \times \pi_{m}\left(\mathrm{~d} r_{m}\right) \times \mathrm{d} t
$$

for $\omega=\left(r_{1}, \ldots, r_{m}, t\right) \in \mathbb{R}_{+}^{m} \times \mathbb{R}$, and $\pi_{i}, i=1, \ldots, m$, are probability measures on $\mathbb{R}_{+}$.

(b) In particular, the results hold for stationary renewal shot noise processes. Such a process has the structure of a Poisson shot noise process; for more details we refer the reader to [15]. 
The extremal behavior of heavy-tailed renewal shot noise processes with strictly decreasing kernel functions $f:[0,1] \rightarrow[0,1]$ and positive jump sizes has been thoroughly investigated in [26].

\subsection{Examples}

Example 4.1. (Discrete-time MA process.) Let $\xi=\left\{\xi_{k}\right\}_{k \in \mathbb{Z}}$ be an i.i.d. sequence of RVs that are regularly varying in the sense of (2.1) with measure $\sigma$ given by (1.4), and let $\left\{c_{k}\right\}_{k \in \mathbb{Z}}$ be a sequence in $\mathbb{R}$. Define the discrete-time MA process $Y_{n}=\sum_{k=-\infty}^{\infty} c_{n-k} \xi_{k}, n \in \mathbb{Z}$. Suppose that $\sum_{k=-\infty}^{\infty}\left|c_{k}\right|^{\delta}<\infty$ for $\delta<\alpha$ and $\delta \leq 2$, with either $\delta<1$ or both $\alpha>1$ and $\mathrm{E}\left[\xi_{k}\right]=0$. This class includes MA processes with the long-memory property. By [27, Lemma A.3], $Y$ is a stationary process whose one-dimensional marginal distribution is regularly varying with

$$
\lim _{n \rightarrow \infty} n \mathrm{P}\left(Y_{k}>a_{n} x\right)=x^{-\alpha}\left[p \sum_{k=-\infty}^{\infty}\left(c_{k}^{+}\right)^{\alpha}+q \sum_{k=-\infty}^{\infty}\left(c_{k}^{-}\right)^{\alpha}\right] .
$$

As in the proof of Theorem 4.1, for $i_{1}, \ldots, i_{d} \in \mathbb{N}$ and as $n \rightarrow \infty$ we have

$$
\sum_{k=1}^{\infty} \varepsilon_{\left(k / n, a_{n}^{-1}\left(Y_{k}, Y_{k+i_{1}}, \ldots, Y_{k+i_{d}}\right)\right)} \stackrel{\mathrm{w}}{\rightarrow} \sum_{k=1}^{\infty} \sum_{j=-\infty}^{\infty} \varepsilon_{\left(s_{k},\left(c_{j}, c_{j-i_{1}}, \ldots, c_{j-i_{d}}\right) P_{k}\right)}
$$

This is a supplement of the well-known result [13, Theorem 2.4] in the case of long-memory processes (see [31] for the case of stable MA processes).

Example 4.2. (Continuation of Example 3.1.) We investigate the extremal behavior of a separable, measurable, and stationary version of the supOU process $Y$ given by (3.4) and driven by an IDISRM $\Lambda$ with generating quadruple $\left(m, \sigma^{2}, v, \pi\right)$, where $\int_{\mathbb{R}_{+}} r^{-1} \pi(\mathrm{d} r)<\infty$ and $\left(m, \sigma^{2}, v\right)$ is the generating triplet of the underlying driving Lévy process $L$, as given in (1.2), which satisfies Condition 1.1 with $p>0$. Let $0=t_{1}<\cdots<t_{d}$. Then, by Theorem 4.1,

$$
\sum_{k=1}^{\infty} \varepsilon_{\left(\Gamma_{k} / T,\left\{Y\left(\Gamma_{k}+t_{i}\right) / a_{\lfloor T\rfloor}\right\}_{i=1, \ldots, d}\right)} \stackrel{\mathrm{W}}{\rightarrow} \sum_{k=1}^{\infty} \sum_{j=0}^{\infty} \varepsilon_{\left(s_{k},\left\{\exp \left(-R_{k}\left(T_{k, j}+t_{i}\right)\right) \chi_{k} P_{k}\right\}_{i=1, \ldots, d}\right)}
$$

holds as $T \rightarrow \infty$. If $Y$ has an exceedance over a high level on the discrete-time skeleton $\left\{\Gamma_{k}+t_{i}, k \in \mathbb{N}, i=1, \ldots, d\right\}$, then we have an extreme at $Y\left(\Gamma_{k}\right)$ for some $k \in \mathbb{N}$. Furthermore, if $Y$ has a.s. sample paths in $\mathbb{D}(\mathbb{R})$, then $\int_{-\infty}^{\infty}(1 \wedge|x|) v(\mathrm{~d} x)<\infty$. By Theorem 4.2 , the running maxima are in the maximum domain of attraction of the Fréchet distribution with

$$
\lim _{T \rightarrow \infty} \mathrm{P}\left(a_{\lfloor T\rfloor}^{-1} M(T) \leq x\right)=\exp \left(-p x^{-\alpha}\right) \quad \text { for } x>0 .
$$

\section{Proofs}

\subsection{Proof of Lemma 2.2}

Let $\epsilon>0$. Denote by $\zeta_{n}:=\sum_{k=1}^{\infty} \varepsilon_{\left(k / n, a_{n}^{-1}\left(\boldsymbol{Z}_{k}+\boldsymbol{\Psi}_{k}\right)\right)}, n \in \mathbb{N}$, a point process in $M_{\mathrm{P}}(S)$. Define the sets

$$
I_{\epsilon}^{(1)}=\prod_{i=1}^{d}\left(c_{i}-\epsilon, d_{i}+\epsilon\right], \quad I_{\epsilon}^{(2)}=\prod_{i=1}^{d}\left(c_{i}+\epsilon, d_{i}-\epsilon\right], \quad \text { and } \quad I_{\epsilon}=I_{\epsilon}^{(1)} \backslash I_{\epsilon}^{(2)} .
$$


We can show that

$$
\begin{gathered}
\left\{\tilde{\kappa}_{n}(I) \neq \zeta_{n}(I)\right\} \subseteq\left\{\tilde{\kappa}_{n}\left(I_{\epsilon}\right)>0\right\} \cup \bigcup_{k \in(n s, n t]}\left\{a_{n}^{-1}\left(\boldsymbol{Z}_{k}+\boldsymbol{\Psi}_{k}\right) \in I, a_{n}^{-1} \boldsymbol{Z}_{k} \in I_{\epsilon}^{(1) \mathrm{c}}\right\} \\
\cup \bigcup_{k \in(n s, n t]}\left\{a_{n}^{-1}\left(\boldsymbol{Z}_{k}+\boldsymbol{\Psi}_{k}\right) \in I^{\mathrm{c}}, a_{n}^{-1} \boldsymbol{Z}_{k} \in I_{\epsilon}^{(2)}\right\} .
\end{gathered}
$$

On the one hand,

$$
\begin{aligned}
\sum_{k \in(n s, n t]} \mathrm{P}\left(a_{n}^{-1}\left(\boldsymbol{Z}_{k}+\boldsymbol{\Psi}_{k}\right) \in I, a_{n}^{-1} \boldsymbol{Z}_{k} \in I_{\epsilon}^{(1) \mathrm{c}}\right) & \leq \sum_{k \in(n s, n t]} \mathrm{P}\left(\left|\boldsymbol{\Psi}_{k}\right|>a_{n} \epsilon\right) \\
& \rightarrow 0, \quad n \rightarrow \infty, \\
\sum_{k \in(n s, n t]} \mathrm{P}\left(a_{n}^{-1}\left(\boldsymbol{Z}_{k}+\boldsymbol{\Psi}_{k}\right) \in I^{\mathrm{c}}, a_{n}^{-1} \boldsymbol{Z}_{k} \in I_{\epsilon}^{(2)}\right) & \leq \sum_{k \in(n s, n t]} \mathrm{P}\left(\left|\boldsymbol{\Psi}_{k}\right|>a_{n} \epsilon\right) \\
& \rightarrow 0, \quad n \rightarrow \infty,
\end{aligned}
$$

and, on the other hand,

$$
\lim _{\epsilon \downarrow 0} \lim _{n \rightarrow \infty} \mathrm{P}\left(\tilde{\kappa}_{n}\left(I_{\epsilon}\right)>0\right)=\lim _{\epsilon \downarrow 0} \mathrm{P}\left(\kappa\left(I_{\epsilon}\right)>0\right)=0 .
$$

Thus, by (5.1)-(5.4) we obtain

$$
\lim _{n \rightarrow \infty} \mathrm{P}\left(\tilde{\kappa}_{n}(I) \neq \zeta_{n}(I)\right)=0 .
$$

After applying [32, Lemma 3.3], we conclude that $\zeta_{n} \stackrel{\mathrm{w}}{\rightarrow} \kappa$ as $n \rightarrow \infty$. A modification of an argument of Hsing and Teugels [19] (the proofs of their Theorem 4.2 and Lemma 2.1; see also [15, Lemma 1.2.4]) yields

$$
\lim _{T \rightarrow \infty} \mathrm{P}\left(\zeta_{\lfloor T\rfloor}(I) \neq \kappa_{T}(I)\right)=0 .
$$

Hence,

$$
\lim _{T \rightarrow \infty} \mathrm{P}\left(\kappa_{T}(I) \neq \tilde{\kappa}_{\lfloor T\rfloor}(I)\right) \leq \lim _{T \rightarrow \infty} \mathrm{P}\left(\kappa_{T}(I) \neq \zeta_{\lfloor T\rfloor}(I)\right)+\lim _{T \rightarrow \infty} \mathrm{P}\left(\zeta_{\lfloor T\rfloor}(I) \neq \tilde{\kappa}_{\lfloor T\rfloor}(I)\right)=0 .
$$

\subsection{Proof of Proposition 3.2}

By [29, Theorem 2.7], the Lévy measure of $Y$ is

$$
\begin{aligned}
v_{Y}(x, \infty)= & \int_{\{(r, s): f(r, s)>0\}} v\left(\frac{x}{f(r, s)}, \infty\right) \mathrm{d} s \pi(\mathrm{d} r) \\
& +\int_{\{(r, s): f(r, s)<0\}} v\left(-\infty, \frac{x}{f(r, s)}\right) \mathrm{d} s \pi(\mathrm{d} r)
\end{aligned}
$$

for $x>0$. By Potter's theorem (see [6, Theorem 1.5.6, p. 25]), for every $x>0$ and $K>1$ there exists an $n_{0}(x) \in \mathbb{N}$ such that $v\left(a_{n} x y, \infty\right) / v\left(a_{n} x, \infty\right) \leq K y^{-\delta}$ for $y \geq 1$ and $n \geq n_{0}$. Since $f \in \mathbb{L}^{\delta}(\pi)$, by dominated convergence and the boundedness of $f$ we find that

$$
\lim _{x \rightarrow \infty} \frac{v_{Y}(x, \infty)}{v(x, \infty)}=\int_{\mathbb{R}_{+}} \int_{\mathbb{R}}\left(p\left(f^{+}(r, s)\right)^{\alpha}+q\left(f^{-}(r, s)\right)^{\alpha}\right) \mathrm{d} s \pi(\mathrm{d} r)
$$

as $n \rightarrow \infty$. Result (3.1) then follows by the tail-equivalence of Lévy measure and probability measure for regularly varying DFs. An application of [33, Theorem 3.1] (cf. [16, Theorem 4.9]) and arguments similar to those above yield (3.2) and (3.3). 


\subsection{Proof of Theorem 4.1}

Step 1. We study the extremal behavior of

$$
\tilde{\boldsymbol{Y}}_{k}^{(m)}=\sum_{j=k-m}^{k+m} \boldsymbol{f}\left(R_{j}, T_{k}-T_{j}\right) Z_{j}, \quad k \in \mathbb{Z},
$$

for $m>0$ fixed, and show that $\left\{\tilde{\boldsymbol{Y}}_{k}^{(m)}\right\}_{k \in \mathbb{Z}}$ satisfies the assumptions of [12, Theorem 2.8 and Corollary 2.4].

We apply Lemma 2.1 to obtain

$$
\lim _{n \rightarrow \infty} n \mathrm{P}\left(\left|\tilde{\boldsymbol{Y}}_{k}^{(m)}\right|>a_{n}\right)=\frac{1}{\mu} \sum_{j=-m}^{m} \mathrm{E}\left[\left|\boldsymbol{f}\left(R_{1}, T_{j}\right)\right|^{\alpha}\right]=: \rho_{m}
$$

Observing that $\left\{\tilde{\boldsymbol{Y}}_{k}^{(m)}\right\}_{k \in \mathbb{Z}}$ is $(2 m+1)$-dependent and taking [23, Lemma 2.4.2] into account, the mixing condition $\mathcal{A}\left(a_{n} \rho_{m}^{1 / \alpha}\right)$ (see [12, p. 2052]) holds for $\left\{\tilde{\boldsymbol{Y}}_{k}^{(m)}\right\}_{k \in \mathbb{Z}}$, i.e. there exists a set of positive integers $\left\{r_{n}\right\}_{n \in \mathbb{N}}$ such that $r_{n} \rightarrow \infty, r_{n} / n \rightarrow 0$ as $n \rightarrow \infty$, and

$$
\mathrm{E}\left[\exp \left(-\sum_{j=1}^{n} f\left(\frac{\tilde{\boldsymbol{Y}}_{j}^{(m)}}{a_{n}}\right)\right)\right]-\left(\mathrm{E} \exp \left[\left(-\sum_{j=1}^{r_{n}} f\left(\frac{\tilde{\boldsymbol{Y}}_{j}^{(m)}}{a_{n}}\right)\right)\right]\right)^{\left\lfloor n / r_{n}\right\rfloor} \rightarrow 0, \quad n \rightarrow \infty
$$

for every bounded, nonnegative step function $f$ on $\overline{\mathbb{R}}^{d} \backslash\{\boldsymbol{0}\}$ with bounded support.

Also, by the $(2 m+1)$-dependence of $\left\{\tilde{\boldsymbol{Y}}_{k}^{(m)}\right\}_{k \in \mathbb{Z}},(5.5)$, and the fact that $r_{n}=o(n)$ as $n \rightarrow \infty$, for $l>2 m+1$ we obtain

$$
\mathrm{P}\left(\bigvee_{l \leq|k| \leq r_{n}}\left|\tilde{\boldsymbol{Y}}_{k}^{(m)}\right|>a_{n} x|| \tilde{\boldsymbol{Y}}_{0}^{(m)} \mid>a_{n} x\right) \leq r_{n} \mathrm{P}\left(\left|\tilde{\boldsymbol{Y}}_{k}^{(m)}\right|>a_{n} x\right) \rightarrow 0, \quad n \rightarrow \infty
$$

Define the random vectors $\boldsymbol{Z}^{(l)}=\left(Z_{-l-m}, \ldots, Z_{l+m}\right)^{\top} \in \mathbb{R}^{2(l+m)+1}, l \in \mathbb{N}$, and the ran$\operatorname{dom}(2 l+1) d \times(2(l+m)+1)$ matrices

$$
\boldsymbol{A}^{(l)}=\left(\begin{array}{c}
\boldsymbol{A}_{-l}^{(l)}, \\
\vdots \\
\boldsymbol{A}_{l}^{(l)}
\end{array}\right),
$$

where $\boldsymbol{A}_{k}^{(l)}, k=-l, \ldots, l$, is a $d \times(2(l+m)+1)$ matrix with $(i, j)$ th entry

$$
\left(\boldsymbol{A}_{k}^{(l)}\right)_{i, j}=f\left(R_{j}, T_{k}-T_{j}+t_{i}\right), \quad i=1, \ldots, d, j=k-m, \ldots, k+m, k=-l, \ldots, l,
$$

and $t_{d}=\eta^{(1)}$. Furthermore,

$$
\left(A_{k}^{(l)}\right)_{i, j}=0, \quad|k-j|>m, i=1, \ldots, d, j=-l-m, \ldots, l+m, k=-l, \ldots, l .
$$

Thus, we have

$$
\left(\tilde{\boldsymbol{Y}}_{-l}^{(m)}, \ldots, \tilde{\boldsymbol{Y}}_{l}^{(m)}\right)^{\top}=\boldsymbol{A}^{(l)} \boldsymbol{Z}^{(l)} \in \mathbb{R}^{(2 l+1) d} .
$$

The matrix $\boldsymbol{A}^{(l)}$ has at most $2 m+1$ entries in a row and $d(2 m+1)$ in a column and the sequence of random matrices $\left\{\boldsymbol{A}_{k}^{(l)}\right\}_{k=-l, \ldots, l}$ is $(2 m+1)$-dependent. 
Since

$$
f^{+} \leq\left\|\boldsymbol{A}^{(l)}\right\| \leq(2 m+1) f^{+},
$$

we can apply Lemma 2.1 to conclude that $\left(\tilde{\boldsymbol{Y}}_{-l}^{(m)}, \ldots, \tilde{\boldsymbol{Y}}_{l}^{(m)}\right)$ is multivariate regularly varying of index $\alpha$ with spectral measure

$$
\mathrm{P}\left(\boldsymbol{\Theta}^{(l)} \in \cdot\right)=\sum_{j=-l-m}^{l+m} \frac{p}{\mu \tilde{\rho}_{m}} \mathrm{E}\left[\left|\boldsymbol{a}_{j}^{(l)}\right|^{\alpha} 1_{\left\{\boldsymbol{a}_{j}^{(l)} /\left|\boldsymbol{a}_{j}^{(l)}\right| \in \cdot\right\}}\right]+\frac{q}{\mu \tilde{\rho}_{m}} \mathrm{E}\left[\left|\boldsymbol{a}_{j}^{(l)}\right|^{\alpha} 1_{\left\{-\boldsymbol{a}_{j}^{(l)} /\left|\boldsymbol{a}_{j}^{(l)}\right| \in \cdot\right\}}\right],
$$

where $\boldsymbol{a}_{j}^{(l)}=\boldsymbol{A}^{(l)} \boldsymbol{e}_{j}, \boldsymbol{e}_{j}=(0, \ldots, 0,1,0, \ldots, 0)^{\top} \in \mathbb{R}^{2(l+m)+1}$ with $j$ th component ' 1 ', and $\tilde{\rho}_{m}:=\mu^{-1} \sum_{j=-l-m}^{l+m} \mathrm{E}\left[\left|\boldsymbol{a}_{j}^{(l)}\right|^{\alpha}\right]$. Furthermore, let $\boldsymbol{\Theta}^{(l)}=\left(\theta_{-1}^{(l)}, \ldots, \Theta_{l}^{(l)}\right)$. Therefore, by $\mathcal{A}\left(a_{n} \rho_{m}^{1 / \alpha}\right),(5.6),(5.7)$, and [12, Theorem 2.8], the point processes $\sum_{k=1}^{n} \varepsilon_{\left(\tilde{\boldsymbol{Y}}_{k}^{(m)} / a_{n}\right)}$ converge weakly to a point process as $n \rightarrow \infty$. In the following, we shall derive the explicit representation of the limit. To obtain the limit distribution, we must compute $\theta_{m}$ and $\mathcal{Q}$, where

$$
\begin{gathered}
\theta_{m}=\lim _{l \rightarrow \infty} \mathrm{E}\left[\left|\Theta_{0}^{(l)}\right|^{\alpha}-\bigvee_{j=1}^{l}\left|\Theta_{j}^{(l)}\right|^{\alpha}\right]^{+}\left(\mathrm{E}\left[\left|\Theta_{0}^{(l)}\right|^{\alpha}\right]\right)^{-1}, \\
\frac{\mathrm{E}\left[\left(\left|\Theta_{0}^{(l)}\right|^{\alpha}-\bigvee_{j=1}^{l}\left|\Theta_{j}^{(l)}\right|^{\alpha}\right)^{+} 1\left(\sum_{|j| \leq l} \varepsilon_{\left(\Theta_{j}^{(l)}\right)} \in \cdot\right)\right]}{\mathrm{E}\left[\left|\Theta_{0}^{(l)}\right|^{\alpha}-\bigvee_{j=1}^{l}\left|\Theta_{j}^{(l)}\right|^{\alpha}\right]^{+}} \stackrel{\mathrm{w}}{\rightarrow} \mathcal{Q}(\cdot), \quad l \rightarrow \infty,
\end{gathered}
$$

so that we can apply [12, Corollary 2.4].

We first derive (5.8). Let $l>2 m+1$. For $j=-m, \ldots, m$ we have

$\bigvee_{k=0}^{l} \bigvee_{i=1}^{d}\left|\left(\boldsymbol{A}_{k}^{(l)}\right)_{i, j}\right|^{\alpha}-\bigvee_{k=1}^{l} \bigvee_{i=1}^{d}\left|\left(\boldsymbol{A}_{k}^{(l)}\right)_{i, j}\right|^{\alpha}=\bigvee_{k=0}^{j+m}\left|\boldsymbol{f}\left(R_{j}, T_{k}-T_{j}\right)\right|^{\alpha}-\bigvee_{k=1}^{j+m}\left|\boldsymbol{f}\left(R_{j}, T_{k}-T_{j}\right)\right|^{\alpha}$,

and for $m<|j| \leq l+m$ we have

$$
\bigvee_{k=0}^{l} \bigvee_{i=1}^{d}\left|\left(\boldsymbol{A}_{k}^{(l)}\right)_{i, j}\right|^{\alpha}-\bigvee_{k=1}^{l} \bigvee_{i=1}^{d}\left|\left(\boldsymbol{A}_{k}^{(l)}\right)_{i, j}\right|^{\alpha}=0
$$

Furthermore, for $j=-m, \ldots, m$ we have

$$
\bigvee_{k=-l}^{l} \bigvee_{i=1}^{d}\left|\left(\boldsymbol{A}_{k}^{(l)}\right)_{i, j}\right|^{\alpha}=\left|\boldsymbol{a}_{j}^{(l)}\right|^{\alpha}=\bigvee_{k=j-m}^{j+m}\left|\boldsymbol{f}\left(R_{j}, T_{k}-T_{j}\right)\right|^{\alpha}=\left(f^{+}\right)^{\alpha} .
$$

By taking the conditional probability under $\Gamma_{k}$ and $R_{k}, k=-l-m, \ldots, l+m$ (and recalling Remark 2.1), we can calculate with deterministic variables. From (5.7) we obtain

$$
\begin{aligned}
& \mathrm{E}\left[\bigvee_{k=0}^{l}\left|\Theta_{k}^{(l)}\right|^{\alpha}-\bigvee_{k=1}^{l}\left|\Theta_{k}^{(l)}\right|^{\alpha}\right] \\
& \quad=\frac{1}{\mu \tilde{\rho}_{m}} \sum_{j=-l-m}^{l+m} \mathrm{E}\left[\left|\boldsymbol{a}_{j}^{(l)}\right|^{\alpha}\left(\bigvee_{k=0}^{l} \bigvee_{i=1}^{d} \frac{\left|\left(\boldsymbol{A}_{k}^{(l)}\right)_{i, j}\right|^{\alpha}}{\left|\boldsymbol{a}_{j}^{(l)}\right|^{\alpha}}-\bigvee_{k=1}^{l} \bigvee_{i=1}^{d} \frac{\left|\left(\boldsymbol{A}_{k}^{(l)}\right)_{i, j}\right|^{\alpha}}{\left|\boldsymbol{a}_{j}^{(l)}\right|^{\alpha}}\right)\right]
\end{aligned}
$$


By taking (5.10) and (5.11) into account, we find that the right-hand side is equal to

$$
\begin{aligned}
\frac{1}{\mu \tilde{\rho}_{m}} & \left(\sum_{j=-m}^{m} \mathrm{E}\left[\bigvee_{k=0}^{j+m}\left|\boldsymbol{f}\left(R_{j}, T_{k}-T_{j}\right)\right|^{\alpha}\right]-\sum_{j=-m+1}^{m} \mathrm{E}\left[\bigvee_{k=1}^{j+m}\left|\boldsymbol{f}\left(R_{j}, T_{k}-T_{j}\right)\right|^{\alpha}\right]\right) \\
& =\frac{1}{\mu \tilde{\rho}_{m}} \mathrm{E}\left[\bigvee_{k=-m}^{m}\left|\boldsymbol{f}\left(R_{1}, T_{k}\right)\right|^{\alpha}\right] \\
& =\frac{\left(f^{+}\right)^{\alpha}}{\mu \tilde{\rho}_{m}}
\end{aligned}
$$

Similarly, since $\bigvee_{i=1}^{d}\left|\left(\boldsymbol{A}_{0}^{(l)}\right)_{i, j}\right|^{\alpha}=0$ for $|j|>m$, by (5.5) we have

$$
\begin{aligned}
\mathrm{E}\left[\left|\Theta_{0}^{(l)}\right|^{\alpha}\right] & =\frac{1}{\mu \tilde{\rho}_{m}} \mathrm{E}\left[\sum_{j=-l-m}^{l+m}\left|\boldsymbol{a}_{j}^{(l)}\right|^{\alpha} \bigvee_{i=1}^{d} \frac{\left|\left(\boldsymbol{A}_{0}^{(l)}\right)_{i, j}\right|^{\alpha}}{\left|\boldsymbol{a}_{j}^{(l)}\right|^{\alpha}}\right] \\
& =\frac{1}{\mu \tilde{\rho}_{m}} \mathrm{E}\left[\sum_{j=-m}^{m}\left|\boldsymbol{f}\left(R_{1}, T_{j}\right)\right|^{\alpha}\right] \\
& =\frac{1}{\mu \tilde{\rho}_{m}} \mu \rho_{m}=\frac{\rho_{m}}{\tilde{\rho}_{m}} .
\end{aligned}
$$

By applying (5.13) and (5.14), for the extremal index of $\left\{\left|\tilde{\boldsymbol{Y}}_{k}^{(m)}\right|\right\}_{k \in \mathbb{Z}}$ in (5.8) we obtain

$$
\theta_{m}=\left(f^{+}\right)^{\alpha} /\left(\mu \rho_{m}\right) \text {. }
$$

We shall now compute $\mathcal{Q}$. By following the reasoning leading to (5.13) and taking $l>2 m+1$ and (5.12) into account, for $j=-m, \ldots, m$ we obtain

$$
\begin{gathered}
\mathrm{E}\left[\left|\boldsymbol{a}_{j}^{(l)}\right|^{\alpha}\left(\bigvee_{k=0}^{l} \bigvee_{i=1}^{d} \frac{\left|\left(\boldsymbol{A}_{k}^{(l)}\right)_{i, j}\right|^{\alpha}}{\left|\boldsymbol{a}_{j}^{(l)}\right|^{\alpha}} 1\left(\sum_{|k| \leq l} \varepsilon_{\left(\left(\boldsymbol{A}_{k}^{(l)}\right)_{i, j} /\left|\boldsymbol{a}_{j}^{(l)}\right|\right)_{i=1, \ldots, d}} \in\right)\right)\right] \\
=\mathrm{E}\left[\bigvee_{k=-j}^{m}\left|\boldsymbol{f}\left(R_{1}, T_{k}\right)\right|^{\alpha} 1\left(\sum_{|k| \leq m} \varepsilon_{\left(\boldsymbol{f}\left(R_{1}, T_{k}\right) / f^{+}\right)} \in \cdot\right)\right] .
\end{gathered}
$$

Then, in analogy to (5.13), we obtain

$$
\begin{aligned}
& \mathrm{E}\left[\left(\left|\Theta_{0}^{(l)}\right|^{\alpha}-\bigvee_{j=1}^{l}\left|\Theta_{j}^{(l)}\right|^{\alpha}\right)^{+} 1\left(\sum_{|j| \leq l} \varepsilon_{\Theta_{j}^{(l)}} \in\right)\right] \\
& =\frac{\left(f^{+}\right)^{\alpha}}{\tilde{\rho}_{m}}\left(\frac{p}{\mu} \mathrm{E}\left[1\left(\sum_{|j| \leq m} \varepsilon_{\left(f\left(R_{1}, T_{j}\right) / f^{+}\right)} \in\right)\right]+\frac{q}{\mu} \mathrm{E}\left[1\left(\sum_{|j| \leq m} \varepsilon_{\left(-f\left(R_{1}, T_{j}\right) / f^{+}\right)} \in\right)\right]\right) \\
& =\frac{\left(f^{+}\right)^{\alpha}}{\mu \tilde{\rho}_{m}} \mathrm{E}\left[1\left(\sum_{|j|<m} \varepsilon_{\left(f\left(R_{1}, T_{j}\right) \chi_{1} / f^{+}\right)} \in\right)\right] .
\end{aligned}
$$

Hence, by (5.13) and (5.16), the measure $\mathcal{Q}$ of (5.9) is defined by

$$
\mathcal{Q}(\cdot)=\mathrm{P}\left(\sum_{j=-m}^{m} \varepsilon_{\left(f\left(R_{1}, T_{j}\right) \chi_{1} / f^{+}\right)} \in \cdot\right) .
$$


Now let

$$
\tilde{\vartheta}(\mathrm{d} x)=\theta_{m} \alpha x^{-\alpha-1} 1_{(0, \infty)}(x) \mathrm{d} x=\frac{\alpha\left(f^{+}\right)^{\alpha}}{\mu \rho_{m}} x^{-\alpha-1} 1_{(0, \infty)}(x) \mathrm{d} x,
$$

where $\theta_{m}$ is as given in (5.15). By taking (5.5) into account and applying [12, Theorem 2.8 and Corollary 2.4], as $n \rightarrow \infty$ we obtain

$$
\sum_{k=1}^{n} \varepsilon_{\left(\rho_{m}^{1 / \alpha} \tilde{\boldsymbol{Y}}_{k}^{(m)} / a_{n}\right)} \stackrel{\mathrm{W}}{\rightarrow} \sum_{k=1}^{\infty} \sum_{j=-m}^{m} \varepsilon_{\left(\boldsymbol{f}\left(R_{k}, T_{k, j}\right) \chi_{k} \tilde{P}_{k} / f^{+}\right)},
$$

where $\sum_{k=1}^{\infty} \varepsilon_{\tilde{P}_{k}}$ is a $\operatorname{PRM}(\tilde{\vartheta})$ in $M_{\mathrm{P}}\left(\overline{\mathbb{R}}^{d} \backslash\{\boldsymbol{0}\}\right)$. By [18, Lemma 4.1.2], the convergence of the sequence of point processes $\kappa_{n}((0,1] \times \cdot)$ is equivalent to the convergence of $\kappa_{n}$ if the so-called $\Delta\left(a_{n}\right)$ condition, which is similar to condition $\mathcal{A}\left(a_{n}\right)$, is satisfied. Note that, by the $(2 m+1)$-dependence of $\left\{\tilde{\boldsymbol{Y}}_{k}^{(m)}\right\}_{k \in \mathbb{Z}}$, the $\Delta\left(a_{n}\right)$ condition holds. This implies, upon replacement of $\tilde{\vartheta}$ by $\vartheta$ and $\left\{\tilde{P}_{k}\right\}_{k \in \mathbb{N}}$ by $\left\{P_{k}\right\}_{k \in \mathbb{N}}$, that

$$
\sum_{k=1}^{\infty} \varepsilon_{\left(k /(n \mu), \tilde{Y}_{k}^{(m)} / a_{n}\right)} \stackrel{\mathrm{w}}{\rightarrow} \sum_{k=1}^{\infty} \sum_{j=-m}^{m} \varepsilon_{\left(s_{k}, f\left(R_{k}, T_{k, j}\right) \chi_{k} P_{k}\right), \quad n \rightarrow \infty .}
$$

Step 2. For a fixed $m>0$, we study the extremal behavior of

$$
\boldsymbol{Y}_{k}^{(m)}=\sum_{j=k-m}^{k+m} \boldsymbol{f}\left(R_{j}, \Gamma_{k}-\Gamma_{j}\right) Z_{j}, \quad k \in \mathbb{Z} .
$$

Note that $\boldsymbol{f}\left(R_{j}, T_{k}-T_{j}\right)=\boldsymbol{f}\left(R_{j}, \Gamma_{k+1}-\Gamma_{j+1}\right)$ for $k, j \in \mathbb{N}_{0}$, by (4.3). Then we also have

$$
\left\{\tilde{\boldsymbol{Y}}_{k}^{(m)}\right\}_{k \geq m} \stackrel{\mathrm{D}}{=}\left\{\boldsymbol{Y}_{k+1}^{(m)}\right\}_{k \geq m},
$$

although $\left\{\boldsymbol{Y}_{k+1}^{(m)}\right\}_{k \in \mathbb{Z}}$ is not stationary. Thus, the asymptotic point process behaviors of $\left\{\tilde{\boldsymbol{Y}}_{k}^{(m)}\right\}_{k \in \mathbb{N}}$ and $\left\{\boldsymbol{Y}_{k}^{(m)}\right\}_{k \in \mathbb{N}}$ are the same. From (5.17), as $n \rightarrow \infty$ we obtain

$$
\sum_{k=1}^{\infty} \varepsilon_{\left(k /(n \mu), \boldsymbol{Y}_{k}^{(m)} / a_{n}\right)} \stackrel{\mathrm{w}}{\rightarrow} \sum_{k=1}^{\infty} \sum_{j=-m}^{m} \varepsilon_{\left(s_{k}, \boldsymbol{f}\left(R_{k}, T_{k}, j\right) \chi_{k} P_{k}\right)}
$$

Step 3. We now study the extremal behavior of $\left\{\boldsymbol{Y}_{k}\right\}_{k \in \mathbb{Z}}$, where

$$
\boldsymbol{Y}_{k}=\sum_{j=-\infty}^{\infty} f\left(R_{j}, \Gamma_{k}-\Gamma_{j}\right) Z_{j}
$$

We must consider the nonstationarity of the sequences $\left\{\boldsymbol{Y}_{k}\right\}_{k \in \mathbb{Z}},\left\{\boldsymbol{Y}_{k}^{(m)}\right\}_{k \in \mathbb{Z}}$, and $\left\{\boldsymbol{Y}_{k}-\boldsymbol{Y}_{k}^{(m)}\right\}_{k \in \mathbb{Z}}$. From (4.3), we have

$$
\begin{aligned}
\mathrm{P}\left(\bigvee_{k=1}^{n}\left|\boldsymbol{Y}_{k}-\boldsymbol{Y}_{k}^{(m)}\right|>a_{n} x\right) \leq & \sum_{i=1}^{d} \sum_{k=1}^{n} \mathrm{P}\left(\left|\sum_{|k-j|>m} f\left(R_{j}, \Gamma_{k}-\Gamma_{j}+t_{i}\right) Z_{j}\right|>a_{n} x\right) \\
\leq & \sum_{i=1}^{d}\left(n \mathrm{P}\left(\left|\sum_{|j| \geq m} f\left(R_{j}, \Gamma_{j}+t_{i}\right) Z_{j}\right|>\frac{a_{n} x}{2}\right)\right. \\
& \left.+\sum_{k=1}^{n} \mathrm{P}\left(\left|f\left(R_{1}, \Gamma_{k}+t_{i}\right) Z_{1}\right|>\frac{a_{n} x}{2}\right)\right) .
\end{aligned}
$$


Since

$$
\sum_{k=1}^{\infty} \mathrm{E}\left[\left|f\left(R_{1}, \Gamma_{k}+t_{i}\right)\right|^{\delta}\right]=\mu \int_{\mathbb{R}_{+}} \int_{\mathbb{R}_{+}}|f(r, s)|^{\delta} \mathrm{d} s \pi(\mathrm{d} r)<\infty,
$$

we see, from Example 2.1(b), that the final term of (5.18) tends to 0. For the first term of (5.18) we have, by a simple generalization of Proposition 3.2,

$$
\begin{aligned}
\lim _{n \rightarrow \infty} n \mathrm{P}\left(\left|\sum_{|j| \geq m} f\left(R_{j}, \Gamma_{j}+t_{i}\right) Z_{j}\right|>\frac{a_{n} x}{2}\right) & =\frac{(x / 2)^{-\alpha}}{\mu} \sum_{|j| \geq m} \mathrm{E}\left[\left|f\left(R_{j}, \Gamma_{j}+t_{i}\right)\right|^{\alpha}\right] \\
& \rightarrow 0 \quad(\text { as } m \rightarrow \infty) .
\end{aligned}
$$

Thus,

$$
\lim _{m \rightarrow \infty} \lim _{n \rightarrow \infty} \mathrm{P}\left(\bigvee_{k=1}^{n}\left|\boldsymbol{Y}_{k}-\boldsymbol{Y}_{k}^{(m)}\right|>a_{n} x\right)=0 .
$$

Then, following the proof of [30, Proposition 4.2.7, p. 236-238], by an argument similar to that in Step 2 we obtain

$$
\sum_{k=1}^{\infty} \varepsilon_{\left(k /(n \mu), \boldsymbol{Y}_{k} / a_{n}\right)} \stackrel{\mathrm{w}}{\rightarrow} \kappa, \quad n \rightarrow \infty .
$$

Step 4. Finally, we study the point process behavior of $\kappa_{n}$.

We invoke the decomposition (4.2), namely $\boldsymbol{Y}(t)=\boldsymbol{Y}_{1}(t)+\boldsymbol{Y}_{2}(t), t \in \mathbb{R}$. Then

$$
\boldsymbol{Y}\left(\Gamma_{k}\right)=\boldsymbol{Y}_{k}+\boldsymbol{Y}_{2}\left(\Gamma_{k}\right) \text { for } k \in \mathbb{Z} .
$$

In analogy to (5.18), we have

$$
\begin{aligned}
\mathrm{P}\left(\left|\boldsymbol{Y}\left(\Gamma_{k}\right)\right|>a_{n} x\right) \leq & \sum_{i=1}^{d} \mathrm{P}\left(\left|\sum_{\substack{j=-\infty \\
j \neq k}}^{\infty} f\left(R_{j}, T_{j}+t_{i}\right) Z_{j}\right|>\frac{a_{n} x}{2}\right) \\
& +\mathrm{P}\left(\left|\boldsymbol{Y}_{2}\left(\Gamma_{k}\right)\right|>a_{n} x / 2\right) .
\end{aligned}
$$

On the one hand, by Proposition 3.2,

$$
\begin{aligned}
& \mathrm{P}\left(\left|\sum_{\substack{j=-\infty \\
j \neq k}}^{\infty} f\left(R_{j}, T_{j}+t_{i}\right) Z_{j}\right|>\frac{a_{n} x}{2}\right) \\
& \quad \leq \mathrm{P}\left(\left|\sum_{j=-\infty}^{\infty} f\left(R_{j}, \Gamma_{j}+t_{i}\right) Z_{j}\right|>\frac{a_{n} x}{6}\right)+2 \mathrm{P}\left(f^{+}\left|Z_{1}\right|>\frac{a_{n} x}{6}\right)=O\left(\frac{1}{n}\right)
\end{aligned}
$$

as $n \rightarrow \infty$. On the other hand, the Lévy measure of $Y_{2}$ has bounded support, meaning that, by [34, Theorem 26.1, p. 168] and the fact that $a_{n} \in \mathcal{R}_{1 / \alpha}$, we have

$$
\mathrm{P}\left(\left|\boldsymbol{Y}_{2}\left(\Gamma_{k}\right)\right|>a_{n} x / 2\right) \leq d \mathrm{P}\left(\left|Y_{2}(0)\right|>a_{n} x / 2\right)=o(1 / n) \quad \text { as } n \rightarrow \infty .
$$

From (5.20)-(5.22), we find that there exists an RV $W$ such that

$$
\mathrm{P}\left(\left|\boldsymbol{Y}\left(\Gamma_{k}\right)\right|>a_{n} x\right) \leq \mathrm{P}\left(W>a_{n} x\right)=O(1 / n) \quad \text { as } n \rightarrow \infty .
$$

Thus, by (5.19), (5.22), Lemma 2.2, and Example 2.1(a), the point process behavior of the sequence $\left\{\boldsymbol{Y}\left(\Gamma_{k}\right)\right\}_{k \in \mathbb{Z}}$ is the same as that of $\left\{\boldsymbol{Y}_{k}\right\}_{k \in \mathbb{Z}}$. Furthermore, we can shift the time-scale. This completes the proof. 


\subsection{Proof of Theorem 4.2}

We first prove part (a). Define the disjoint intervals

$$
I_{k}=\left[\eta^{(1)}+\Gamma_{k}, \eta^{(1)}+\Gamma_{k+1}\right), \quad k \in \mathbb{N} .
$$

Let $\tilde{Y}(t)=\sum_{j=-\infty}^{\infty} \tilde{f}\left(R_{j}, t-\Gamma_{j}\right) Z_{j}^{+}, t \in \mathbb{R}$, be a mixed MA process, which, by Proposition 3.1 , is stationary and ID. Define

$$
\tilde{\boldsymbol{Y}}(t)=\left(\tilde{Y}\left(t+t_{1}\right), \ldots, \tilde{Y}\left(t+t_{d-1}\right), \tilde{Y}\left(t+\eta^{(1)}\right)\right) .
$$

Applying Theorem 4.1 to $\tilde{\boldsymbol{Y}}$ yields, as $T \rightarrow \infty$,

$$
\tilde{\kappa}_{T}:=\sum_{k=1}^{\infty} \varepsilon_{\left(\Gamma_{k} / T, \tilde{\boldsymbol{Y}}\left(\Gamma_{k}\right) / a_{T}\right)} \stackrel{\mathrm{w}}{\rightarrow} \sum_{k=1}^{\infty} \sum_{j=-\infty}^{\infty} \varepsilon_{\left(s_{k}, \tilde{\boldsymbol{f}}\left(R_{k}, T_{k, j}\right) P_{k} \chi_{k}^{+}\right)}=: \tilde{\kappa} .
$$

Moreover, define

$$
\overline{\boldsymbol{Y}}_{k}:=\tilde{\boldsymbol{Y}}\left(\Gamma_{k}\right)+\sup _{s \in I_{k}} \boldsymbol{Y}_{2}(s),
$$

where we understand $\sup _{s \in I_{k}} \boldsymbol{Y}_{2}(s)$ to mean the coordinatewise supremum. Then

$$
\boldsymbol{Y}(t) \leq \overline{\boldsymbol{Y}}_{k} \quad \text { for } t \in I_{k},
$$

again coordinatewise. Keep in mind the fact that

$$
\mathrm{P}\left(\left|\overline{\boldsymbol{Y}}_{k}\right|>a_{n} x\right) \leq \mathrm{P}\left(\left|\tilde{\boldsymbol{Y}}\left(\Gamma_{k}\right)\right|>a_{n} x / 2\right)+\mathrm{P}\left(\sup _{s \in I_{k}}\left|\boldsymbol{Y}_{2}(s)\right|>a_{n} x / 2\right) .
$$

On the one hand, we obtain

$$
\tilde{\boldsymbol{Y}}\left(\Gamma_{k}\right)=\sum_{j=-\infty}^{\infty} \tilde{\boldsymbol{f}}\left(R_{j}, \Gamma_{k}-\Gamma_{j}\right) Z_{j}^{+} \stackrel{\mathrm{D}}{=} \sum_{\substack{j=-\infty \\ j \neq k}}^{\infty} \tilde{\boldsymbol{f}}\left(R_{j}, T_{j}\right) Z_{j}^{+} \leq \sum_{j=-\infty}^{\infty} \tilde{\boldsymbol{f}}\left(R_{j}, T_{j}\right) Z_{j}^{+}
$$

where, by Proposition 3.2 and the independence of $\sum_{j=-\infty, j \neq 0}^{\infty} \tilde{\boldsymbol{f}}\left(R_{j}, T_{j}\right) Z_{j}^{+}$and $\tilde{\boldsymbol{f}}\left(R_{1}, 0\right) Z_{1}^{+}$,

$$
\lim _{n \rightarrow \infty} n \mathrm{P}\left(\left|\sum_{j=-\infty}^{\infty} \tilde{\boldsymbol{f}}\left(R_{j}, T_{j}\right) Z_{j}^{+}\right|>a_{n} x\right)=\frac{p}{\mu x^{\alpha}} \sum_{j=-\infty}^{\infty} \mathrm{E}\left[\left|\tilde{\boldsymbol{f}}\left(R_{1}, T_{j}\right)\right|^{\alpha}\right] .
$$

On the other hand, the Lévy measure of $Y_{2}$ has bounded support. Using the Markov inequality, [7, Lemma 2.1], and the facts that $a_{n} \in \mathcal{R}_{1 / \alpha}$ and $I_{k}$ is independent of $Y_{2}$ yields

$$
\mathrm{P}\left(\sup _{s \in I_{k}}\left|\boldsymbol{Y}_{2}(s)\right|>a_{n} x / 2\right) \leq d(1 / \mu+1) \mathrm{e}^{-a_{n} x / 2} \mathrm{E}\left[\exp \left(\sup _{0 \leq s \leq 1}\left|Y_{2}(s)\right|\right)\right]=o(1 / n)
$$

as $n \rightarrow \infty$. From (5.26)-(5.29), we find that there exists an RV $W$ such that

$$
\mathrm{P}\left(\left|\overline{\boldsymbol{Y}}_{k}\right|>a_{n} x\right) \leq \mathrm{P}\left(W>a_{n} x\right)=O(1 / n) \quad \text { as } n \rightarrow \infty .
$$


Thus, by (5.24), (5.29), Lemma 2.2, and Example 2.1(a), the point process behavior of the sequence $\left\{\overline{\boldsymbol{Y}}_{k}\right\}_{k \in \mathbb{Z}}$ is the same as that of $\left\{\tilde{\boldsymbol{Y}}\left(\Gamma_{k}\right)\right\}_{k \in \mathbb{Z}}$. Furthermore, we can shift the time-scale. This together with (5.23) yields, as $T \rightarrow \infty$,

$$
\tilde{\kappa}_{T}:=\sum_{k=1}^{\infty} \varepsilon_{\left(\Gamma_{k} / T, \overline{\boldsymbol{Y}}_{k} / a_{\lfloor T\rfloor}\right)} \stackrel{\mathrm{W}}{\rightarrow} \tilde{\kappa} .
$$

By taking (5.25) into account, on the one hand, for $I=[0,1) \times \mathbb{R}_{+}^{d} \backslash(0, x]^{d}$ we obtain

$$
\lim _{T \rightarrow \infty} \mathrm{P}\left(a_{\lfloor T\rfloor}^{-1} M(T) \leq x\right) \geq \mathrm{P}(\tilde{\kappa}(I)=0)=\exp \left(-\left(f^{+}\right)^{\alpha} p x^{-\alpha}\right) .
$$

On the other hand, Theorem 4.1 applied to $\boldsymbol{Y}$ yields

$$
\lim _{T \rightarrow \infty} \mathrm{P}\left(a_{\lfloor T\rfloor}^{-1} M(T) \leq x\right) \leq \mathrm{P}(\kappa(I)=0)=\exp \left(-\left(f^{+}\right)^{\alpha} p x^{-\alpha}\right) .
$$

The result follows from (5.30) and (5.31).

The proof of part (b) follows along the lines of the proof of [16, Theorem 5.8], in which the normalizing constants for subexponential Lévy-driven MA processes in the maximum domain of attraction of the Gumbel distribution were calculated. The only difference is that the point process results for regularly varying processes are applied here, in particular Theorem 4.1 and the results for discrete-time MA processes (see Example 4.1).

\section{Acknowledgements}

I take pleasure in thanking my $\mathrm{PhD}$ advisor Claudia Klüppelberg for helpful comments and careful proof-reading. Financial support by the Deutsche Forschungsgemeinschaft through the graduate program in applied algorithmic mathematics at Munich University of Technology is gratefully acknowledged.

\section{References}

[1] Albin, J. M. P. (2000). Extremes and upcrossing intensities for $P$-differentiable stationary processes. Stoch. Process. Appl. 87, 199-234.

[2] Barndorff-Nielsen, O. E. (2001). Superposition of Ornstein-Uhlenbeck type processes. Theory Prob. Appl. 45, 175-194.

[3] Barndorff-Nielsen, O. E. And Shephard, N. (2001). Modelling by Lévy processes for financial econometrics. In Lévy Processes, eds O. E. Barndorff-Nielsen, T. Mikosch and S. I. Resnick, Birkhäuser, Boston, MA, pp. 283318.

[4] Basrak, B., Davis, R. A. And Mikosch, T. (2002). A characterization of multivariate regular variation. Ann. Appl. Prob. 12, 908-920.

[5] Basrak, B., Davis, R. A. And Mikosch, T. (2002). Regular variation of GARCH processes. Stoch. Process. Appl. 99, 95-115.

[6] Bingham, N. H., Goldie, C. M. and Teugels, J. L. (1987). Regular Variation. Cambridge University Press.

[7] Braverman, M. and Samorodnitsky, G. (1995). Functionals of infinitely divisible stochastic processes with exponential tails. Stoch. Process. Appl. 56, 207-231.

[8] Breiman, L. (1965). On some limit theorems similar to the arc-sine law. Theory Prob. Appl. 10, 323-331.

[9] Brockwell, P. J. ANd Marquardt, T. (2005). Lévy-driven and fractionally integrated ARMA processes with continuous time parameter. Statist. Sinica 15, 477-494.

[10] Daley, D. J. And Vere-Jones, D. (2003). An Introduction to the Theory of Point Processes, Vol. 1, Elementary Theory and Methods, 2nd edn. Springer, New York.

[11] Davis, R. AND Hsing, T. (1995). Point process and partial sum convergence for weakly dependent random variables with infinite variance. Ann. Prob. 23, 879-917. 
[12] Davis, R. And Mikosch, T. (1998). The sample autocorrelations of heavy-tailed processes with applications to ARCH. Ann. Statist. 26, 2049-2080.

[13] DAVIS, R. AND Resnick, S. (1985). Limit theory for moving averages of random variables with regularly varying tail probabilities. Ann. Prob. 13, 179-195.

[14] Embrechts, P., Klüppelberg, C. And Mikosch, T. (1997). Modelling Extremal Events for Insurance and Finance. Springer, Berlin.

[15] FASEN, V. (2004). Extremes of Lévy driven MA processes with applications in finance. Doctoral Thesis, Munich University of Technology. Available at http://tumb1.biblio.tu-muenchen.de/publ/diss/ma/2004/fasen.html.

[16] FASEN, V. (2005). Extremes of subexponential Lévy driven moving average processes. Submitted.

[17] Gushchin, A. A. AND Küchler, U. (2000). On stationary solutions of delay differential equations driven by a Lévy process. Stoch. Process. Appl. 88, 195-211.

[18] Hsing, T. (1993). On some estimates based on sample behavior near high level excursions. Prob. Theory Relat. Fields 95, 331-356.

[19] Hsing, T. and Teugels, J. L. (1989). Extremal properties of shot noise processes. Adv. Appl. Prob. 21, 513-525.

[20] Hult, H. And LindskøG, F. (2005). Extremal behavior of regularly varying stochastic processes. Stoch. Process. Appl. 115, 249-274.

[21] Kallenberg, O. (1997). Foundations of Modern Probability. Springer, New York.

[22] Kwapień, S. and Woyczyński, W. A. (1992). Random Series and Stochastic Integrals: Single and Multiple. Birkhäuser, Boston, MA.

[23] Leadbetter, M. R. and Rootzén, H. (1988). Extremal theory for stochastic processes. Ann. Prob. 16, 431-478.

[24] LebedeV, A. V. (2000). Extremes of subexponential shot noise. Math. Notes 71, 206-210.

[25] LindsKøG, F. (2004). Multivariate extremes and regular variation for stochastic processes. Doctoral Thesis, ETH Zürich. Available at http://e-collection.ethbib.ethz.ch/cgi-bin/show.pl?type=diss\&nr=15319.

[26] МсCовміск, W. P. (1997). Extremes for shot noise processes with heavy tailed amplitudes. J. Appl. Prob. 34, 643-656.

[27] Mikosch, T. and Samorodnitsky, G. (2000). The supremum of a negative drift random walk with dependent heavy-tailed steps. Ann. Appl. Prob. 10, 1025-1064.

[28] Pedersen, J. (2003). The Lévy-Ito decomposition of an independently scattered random measure. Tech. Rep. 2003-2, Centre for Mathematical Physics and Stochastics, University of Aarhus. Available at http://www. maphysto.dk/oldpages/publications/publications2003_static.html.

[29] RAJPUT, B. S. AND Rosiński, J. (1989). Spectral representations of infinitely divisible processes. Prob. Theory Relat. Fields 82, 453-487.

[30] Resnick, S. I. (1987). Extreme Values, Regular Variation, and Point Processes. Springer, New York.

[31] Rootzén, H. (1978). Extremes of moving averages of stable processes. Ann. Prob. 6, 847-869.

[32] Rootzén, H. (1986). Extreme value theory for moving average processes. Ann. Prob. 14, 612-652.

[33] Rosiński, J. AND SAmorodnitsky, G. (1993). Distributions of subadditive functionals of sample paths of infinitely divisible processes. Ann. Prob. 21, 996-1014.

[34] Sato, K. (1999). Lévy Processes and Infinitely Divisible Distributions. Cambridge University Press.

[35] Urbanic, K. AND Woyczyński, W. A. (1968). Random measures and harmonizable sequences. Studia Math. 31, 61-88. 